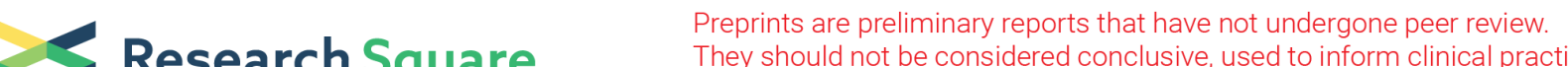 Research Square They should not be considered conclusive, used to inform clinical practice,

\section{KCTD1 Downregulation Suppresses Hepatocellular Carcinoma by Regulating Angiogenesis and Influencing the HIF-1a/VEGF Pathway}

\section{Xinyu Zhu}

Hunan Normal University

\section{Zhiwei Li}

Hunan Normal University

Limin Li

Hunan Normal University

Li Chen

Hunan Normal University

Mi Ouyang

Hunan Normal University

Hao Zhou

Hunan Normal University

Kai Xiao

Xiangya Hospital Central South University

Ling Lin

Xiangya Hospital Central South University

Paul K. Chu

City University of Hong Kong

Chang Zhou

Hunan Normal University

Chengfeng Xun

Hunan Normal University

Liu Yang

Hunan Normal University

Wenhuan Huang

Hunan Normal University

Xiaofeng Ding ( $\nabla$ dingxiaofeng@hunnu.edu.cn )

Hunan Normal University 
Keywords: KCTD1, hepatocellular carcinoma, angiogenesis, VEGF, sorafenib

Posted Date: May 7th, 2021

DOI: https://doi.org/10.21203/rs.3.rs-419484/v1

License: (c) (1) This work is licensed under a Creative Commons Attribution 4.0 International License. Read Full License 


\section{Abstract}

Background: Potassium channel tetramerization domain-containing 1 (KCTD1) plays a critical role in transcriptional regulation and adipogenesis, but the significance of KCTD1 in hepatocellular cancer (HCC) has not been reported.

Methods: KCTD1 expression was detected by immunohistochemistry, Western blotting, and quantitative RT-PCR. Malignant behaviors of cells were analyzed by MTT assays, liquid colony formation, scratch, transwell assays and FACS analysis. The in vivo functional assays were examined by mouse models using subcutaneous, tail vein, intrahepatic injection combined with sorafenib or exosome treatment. The angiogenesis was analyzed by tube formation and Matrigel plug model. The protein-protein interaction was demonstrated by Co-immunoprecipitation. The protein-DNA binding was confirmed by luciferase assays and chromatin immunoprecipitation.

Results: KCTD1 expression is increased in human HCC tissues and closely associated with advanced tumor stages. KCTD1 overexpression enhances HCC growth, migration, and invasion both in vitro and in vivo, while KCTD1 knockdown reverses these effects. Moreover, KCTD1 overexpression promotes the in vitro growth, migration, and tube formation of human umbilical-vein endothelial cells (HUVECs) and induces in vivo angiogenesis. Mechanistically, KCTD1 interacts with HIF-1a to activate the VEGF pathway in HCC cells, which is concurrent with increased M2 macrophage infiltration. Sorafenib blocks the expression of KCTD1 protein and synergistically inhibits subcutaneous and intrahepatic tumor growth. miR-129-5p downregulates KCTD1 expression by binding with KCTD1 3' UTR and abrogates the oncogenic role of KCTD1 in vitro. Finally, exosomes derived from miR-129-5p-overexpressing HCC cells combine with sorafenib to decrease HCC tumor size.

Conclusions: These results suggest that KCTD1 expression activates the VEGF signaling cascade to enhance HCC progression, and angiogenesis. Therefore, KCTD1 may serve as a novel target of sorafenib and pave the way for an efficient therapy for advanced human HCC.

\section{Background}

Hepatocellular cancer (HCC) is a typical hypervascular solid tumor in which angiogenesis plays central roles in development, progression and metastasis [1] and increase in the microvascular density of tumor tissues indicates poor prognosis for patients with HCC [2]. The vascular endothelial growth factor (VEGF) family is a growth factor specific for vascular endothelial cells [3]. The VEGF serves as an angiogenesis biomarker as well as a major target for anti-angiogenic therapy for HCC mainly through the activation of VEGF receptor 2 (VEGFR2) [4]. VEGF-A is ubiquitously expressed in most of tumor cells, and tumor stroma cells, such as fibroblasts, and inflammatory cells, during tumor progression, whereas VEGFR2 is expressed at high levels in tumor-associated endothelial cells resulting in abnormal tumor vasculature [5]. VEGF-A binds to and activates VEGFR2 to promote angiogenesis, vascular permeability, and cell migration in vascular disease and cancer [6]. The kinase inhibitor sorafenib exhibits 
antiproliferative and antiangiogenic properties predominantly through inhibition of Raf-kinase and VEGFR2 and functions as the FDA-approved targeted drug to improve the median overall survival time by about three months for patients with advanced HCC [7]. Therefore, the molecular events underlying $\mathrm{HCC}$ progression and angiogenesis and improved sorafenib sensitivity need to be urgently addressed in order to develop more effective, life-prolonging strategies for future therapeutic interventions of advanced HCC.

The BTB/POZ domain-containing protein KCTD1 has been identified as a transcriptional suppressor and interacts with the AP-2 transcription factor family to inhibit AP-2 transcriptional activities through the BTB domain $[8,9]$. KCTD1 binds to $\beta$-catenin and enhances its degradation by linking casein kinase I

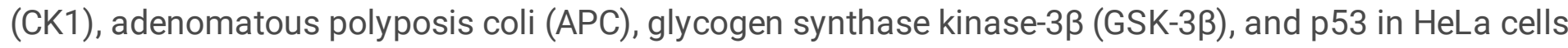
[10]. The physiological significance of KCTD1 has been elucidated and KCTD1 promotes adipogenesis via interactions with AP-2a, a known inhibitor of adipogenesis [11]. The AP-2 $\beta / K C T D 1$ axis blocks renal fibrosis by suppressing $\beta$-catenin activity, while KCTD1 knockout exhibits enhanced renal fibrosis [12]. Mutations found in AP-2a induced aplasia cutis in patients with branchiooculo facial syndrome (BOFS) [13] and mutants in the DNA-binding domain and in a PY motif of the AP-2 $\beta$ gene have been detected from patients with Char syndrome [14], while KCTD1 mutants have been detected from scalpear-nipple (SEN) syndrome $[15,16]$. Our recent studies show that SEN-related KCTD1 mutants and Charrelated AP-2a P59A individually disrupt mutant interactions with the wild-type AP-2a and KCTD1 proteins, but these mutations are able to regulate the $\mathrm{Wnt} / \beta$-catenin pathway [17]. Although only limited roles of KCTD1 have been investigated to date, these findings indicate that KCTD1 is associated with key transcription factors and regulates canonical signaling pathways in development, lipid metabolism and diseases.

Carcinogenesis is a complicated process involving transformation, proliferation, survival, migration, metastasis, angiogenesis, glycolysis and immune evasion, during which multiple genes function and interact [18]. However, there is no reported evidence showing the underlying roles and mechanisms of KCTD1 in carcinogenesis. In this work, the importance of KCTD1 in HCC progression is studied to confirm upregulation of KCTD1 expression in human HCC tissues. The oncogenic effects of KCTD1 are exerted by enhancing the HIF1a/VEGF signaling pathway, which is reversed by the tumor suppressive gene miR-129$5 p$ targeting KCTD1 3' UTR. KCTD1 downregulation sensitizes HCC cells to the sorafenib treatment by inhibiting angiogenesis and decreasing M2 macrophage infiltration. Our results demonstrate that the KCTD1 protein directly interacts with HIF1a to activate the VEGF signaling cascade, thus highlighting KCTD1 as a potential clinical marker in HCC patients and novel target of HCC therapy.

\section{Methods}

\section{Tube-forming Assays}

The HUVEC cells $\left(1 \times 10^{4} /\right.$ well) were grown on a 96 -well plate coated with $50 \mu \mathrm{l}$ of BD matrigel matrix (Bedford, MA, USA) and $150 \mathrm{ng} / \mathrm{ml}$ bFGF in 3\% FBS growth medium or conditioned medium from HCC 
and incubated for $2-6 \mathrm{~h}$ at $37^{\circ} \mathrm{C}$. The wells were then photographed and the capillary-like structures were evaluated and counted from six random fields under an inverted Zeiss Axioskop 2 fluorescence microscope (Carl Zeiss Microscopy, LLC, NY, US). The total tube number and branch count were determined by the ImageJ software.

\section{In vivo Mouse Experiments}

The mouse experiments were performed following the ethical principles and guidelines for Experiments on Animals and approved by Hunan Normal University. The tumorigenic capacity in vivo was determined using the xenograft mouse model. Lentivirus-infected Huh7 $\left(1 \times 10^{7}\right), \mathrm{MHCC} 97 \mathrm{H}\left(8 \times 10^{6}\right)$, and NIH3T3 cells $\left(2 \times 10^{5}\right)$ were suspended in $0.2 \mathrm{~mL}$ of DMEM and subcutaneously injected into two points of the back respectively of BALb/c nude mice (4-week old, female, $n=3-5$ mice per group). The mice were examined every other day and the formed tumors were calculated as stated previously [19]. After two to four weeks, the mice were sacrificed and subcutaneous tumors were removed, photographed and weighed.

The metastatic ability in vivo was determined after intravenous injection of $5 \times 10^{6} \mathrm{Huh} 7$ and $\mathrm{MHCC} 97 \mathrm{H}$ cells into the tail veins of nude mice (6-week old, $n=5$ per group) in two groups. The mice were sacrificed after 5-6 weeks and the number of formed metastasis nodules on the surfaces of mouse lungs and livers were assessed. The lungs and livers were removed, fixed and embedded in paraffin for further hematoxylin and eosin (HE) staining and IHC analysis.

In the 3D Matrigel plug experiments, $5 \times 10^{6}$ lentivirus-infected HUVECs and $5 \times 10^{6}$ Huh7 cells mixed with $250 \mu \mathrm{L}$ of growth-factor reduced Matrigel (BD) and $500 \mathrm{ng} / \mathrm{ml}$ bFGF were subcutaneously injected into the back of BALb/c nude mice (6-week old) to form a single solid gel plug. After the mice were maintained for 10-14 days, the plugs were harvested and photos were taken.

In the mouse intrahepatic experiments, the mice in four groups with 3-5 mice in each group were administered intrahepatic injections of $2 \times 10^{6} \mathrm{MHCC} 97 \mathrm{H}$ cells infected with KCTD1 shRNA. 7 days post injection, the mice were orally gavaged with $25 \mathrm{mg} / \mathrm{kg}$ of sorafenib every 2 days for 15 days or injected three times with $15 \mu \mathrm{g}$ of exosomes per injection via the tail vein for two weeks. After 3 weeks, the mice were then sacrificed and the livers were removed, tumor foci was photographed.

\section{Exosome Extraction}

Exosomes from the cell media were extracted using an exosome isolation kit (Invitrogen, Waltham, USA) following the manufacturer's instructions. Briefly, the fresh cell medium was harvested from $8 \times 10^{6}$ MHCC $97 \mathrm{H}$ cells in a volume of $15 \mathrm{~mL}$ culture medium and grown without FBS for $24 \mathrm{~h}$. The culture medium was centrifuged at $700 \times \mathrm{g}$ for $8 \mathrm{~min}$ to remove the cells and debris. Then, the medium was filtered through $0.22-\mu \mathrm{m}$-pore-size nitrocellulose membrane, the supernatant was added to $7.5 \mathrm{~mL}$ of total exosome isolation reagent and the samples were mixed and incubated overnight at $4^{\circ} \mathrm{C}$. Then, samples were centrifuged at $13,000 \times \mathrm{g}$ at $4^{\circ} \mathrm{C}$ for $70 \mathrm{~min}$. The pellet was resuspended and washed followed by $100,000 \times \mathrm{g}$ centrifugation for $90 \mathrm{~min}$. The pelleted exosomes were finally dissolved in $80 \mu \mathrm{L}$ of PBS and 
stored at $-80^{\circ} \mathrm{C}$. By dropping the exosome suspensions onto an electron microscopy copper grid, the morphology of exosomes were determined by transmission electron microscopy (TEM, JEOL2100, Japan).

\section{Results}

\section{KCTD1 Upregulation in HCC Tissues}

To measure the clinical significance of KCTD1 in malignant tumors, GEPIA based on The Cancer Genome Atlas (TCGA) data is analyzed to examine the expression of KCTD1 across various tumor samples and paired normal tissues. KCTD1 expression is upregulated in most tumor tissues (Fig. 1a) and the HCC database shows that KCTD1 expression increases in HCC tissues when compared with non-tumor liver tissues (Fig. 1b). The expression level of KCTD1 in 10 normal livers and 70 HCCs is detected by IHC analysis. KCTD1 is highly expressed in $50 \%$ of adjacent normal liver tissues and detected in $10(14 \%)$ of the $70 \mathrm{HCCs}$ as indicated by intense staining (3+). 23 (33\%) of the 70 HCCs are moderately positive (2+) and $37(53 \%)$ are weakly positive or negative for KCTD1 expression (1+/0) (Fig. 1c-d). Clinicopathologic association analysis of the $70 \mathrm{HCCs}$ reveals that KCTD1 expression is associated with the advanced clinical stage of HCC (Additional file 1: Table S1). Low KCTD1 expression is observed from low-grade hepatocellular cancers (I/II), whereas high KCTD1 expression is detected from high-grade HCC (III/IV) $(P<0.05)$ (Fig. 1d). In 30 paired HCC and adjacent non-tumor samples, KCTD1 expression shows a high proportion of upregulation in the HCC samples compared with non-tumor samples as analyzed by quantitative RT-PCR (Fig. 1e, Additional file 2: Table S2). Furthermore, high-level KCTD1 expression is correlated negatively with the overall survival of patients according to Kaplan-Meier Plotter database (Fig. 1f) and therefore, KCTD1 expression increases significantly in high-grade HCC tissues (III/IV) compared to adjacent normal liver tissues.

\section{KCTD1 Overexpression Enhances both in vitro and in vivo Proliferation, Migration and Metastasis of HCC Cells}

The effects of KCTD1 overexpression on the malignancy of human HCC are analyzed by examining the expression profile of the KCTD1 protein in the HCC cell lines. The relatively low expression of KCTD1 proteins is detected in human hepatoma cell lines HepG2, Hep3B, and Huh7, while high expression of KCTD1 is found from MHCC97H and SMMC7721 cells (Additional file 3: Figure S1a). The HCC cell lines with low expression and high expression of KCTD1 are selected for KCTD1 overexpression and knockdown, respectively. To evaluate the role of KCTD1 in HCC, full-length KCTD1 is ligated into the pGCFU lentiviral vector and stable expression HCC cell lines (pFLAG-KCTD1 and pFLAG-NC) are generated with HepG2 and Huh7 cells (Figure S1b). Western blotting demonstrates overexpression of KCTD1 in both HCC cell lines (Fig. 2a). KCTD1 overexpression results in more viable HCC cells (Fig. 2b), increased colony number, and larger HCC cell size than the control cells (Fig. 2c and Figure S1c), indicating the strong tumorigenicity of KCTD1. Transwell cell migration assays indicate that overexpression of KCTD1 results in a significant increase in the cell motility (Fig. 2d and Figure S1d). Moreover, transwell Matrigel invasion 
assays reveal that more KCTD1 cells than control cells are invasive (Fig. $2 \mathrm{e}$ and Figure S1e). These results suggest that KCTD1 increases the in vitro proliferation, migration, and invasion of HCC cells.

To corroborate the similar effect of KCTD1 overexpression on the tumorigenic ability of HCC cells in vivo, KCTD1 LV-infected Huh7 cells are injected subcutaneously into the two points of the back of female nude mice, individually. Within 4 weeks, the mean tumor weight and volume in the KCTD1 group increase significantly in comparison with the control group mice (Fig. 2f-g, and Figure S1f-g). HE staining confirms that the HCC cells are arranged tightly in the KCTD1 group (Fig. $2 \mathrm{~h}$ ) and IHC staining shows that KCTD1 overexpression increases the expression of the proliferation marker Ki67, endothelial marker CD31, and M2 macrophage marker Arg1 in the subcutaneous tissues derived from Huh7 cells (Fig. 2i and Figure $\mathrm{S} 1 \mathrm{~h})$. These findings indicate that KCTD1 overexpression enhances the tumorigenic ability of HCC cells in vivo.

KCTD1 overexpressing cells and control Huh7 cells are injected into the tail veins of nude mice (6-week old, female) to check for the lung metastasis, the metastatic nodules on the surfaces of lungs and livers are examined after six weeks (Figure S1i). Tumor foci in the mouse lungs are revealed by HE staining. The mice from the KCTD1 group have larger lung metastatic nodules, while a few smaller nodules are detected from mice with control cells (Fig. 2j). Metastatic nodules also appear from mouse liver tissues obtained from the KCTD1 group. All in all, these findings demonstrate that KCTD1 overexpression promotes the in vivo invasion and metastasis of HCC cells.

To further confirm whether KCTD1 overexpression leads to malignant transformation, we infect NIH3T3 mouse embryonic fibroblast cells with KCTD1 LV and NC LV, examine the infection efficiency by GFP fluorescence (Additional file 4: Figure S2a), and confirm KCTD1 overexpression in the NIH3T3 cells by Western blot analysis (Figure S2b). KCTD1 overexpression enhances the malignant phenotype (Figure S2c) and cell growth (Figure S2d) and induces increased colony formation of the NIH3T3 cells (Figure S2e). The oncogenesis-promoting effect of KCTD1 on tumor formation in vivo is examined. $2 \times 10^{5}$ $\mathrm{NIH} 3 \mathrm{~T} 3$ cells are subcutaneously injected into healthy BALb/c nude female mice and KCTD1overexpressing cells form larger tumors in mice, but the control cells form significantly smaller tumors (Figure S2f-g), suggesting that the oncogenic capabilities of KCTD1 enhance malignant transformation of NIH3T3 cells.

\section{KCTD1 Knockdown suppresses the in vitro and in vivo Proliferation, Migration and Metastasis of HCC Cells}

To confirm the role of KCTD1 in human HCC, KCTD1 shRNA is ligated into the GV248 lentiviral vector (GeneCopoeia) and the stable cell lines (GFP-KCTD1 shRNA and GFP-NC shRNA) are screened and established in MHCC97H and SMMC7721 cells (Additional file 5: Figure S3a). Western blotting demonstrates knockdown of KCTD1 expression in both MHCC97H and SMMC7721 cell lines (Fig. 3a). KCTD1 knockdown decreases the HCC viable cell number (Fig. 3b) and suppresses the number and size of HCC cell colonies (Fig. 3c and Figure S3b). Moreover, the wound-healing assays show that KCTD1 
knockdown suppresses HCC cell migration by about 50\% (Fig. 3d and Figure S3c). Transwell migration assays indicate that knocking down KCTD1 results in significant decrease in the cell motility (Fig. 3e and Figure S3d). Matrigel invasion assays disclose that a significantly smaller proportion of HCC cells with KCTD1 knock down invaded through the Matrigel-coated chamber compared to the number of control HCC cells (Fig. $3 f$ and Figure S3e). The data show a strong antitumorigenic role for KCTD1 downregulation.

The influence of KCTD1 knockdown on the tumorigenicity of HCC cells is examined in vivo. MHCC97H cells stably infected with KCTD1 shRNA-LV or control NC-LV are s.c. injected into the two points of the back of five nude mice, individually and after 2 weeks, the mean tumor weight and volume of the KCTD1knockdown group decrease compared with the NC group (Fig. 3g-h and Figure S3f-g). HE staining confirms that the MHCC97H cells are arranged loosely in the KCTD1 shRNA group (Figure S3h) and IHC analysis shows that KCTD10 knockdown inhibits the expression of Ki67, CD31, and Arg1 (Fig. 3i and Figure S3i). KCTD1 shRNA cells and control MHCC97H cells are intravenously injected via the tail vein into nude mice (6-week old, female) and after six weeks, the metastatic nodules are calculated on the surfaces of mouse lungs and livers (Figure S3j). The mice in the KCTD1 shRNA group have increasingly fewer and smaller lung metastatic nodules than those in the NC shRNA group (Fig. 3j-k). Tumor foci in mouse lungs are detected by HE staining, while the liver morphology does not change obviously in the two groups of mice (Fig. 3l). Altogether, these results demonstrate that KCTD1 downregulation suppresses HCC cell proliferation, migration, and metastasis in vivo, suggesting that KCTD1 knockdown markedly suppresses the tumorigenic ability of HCC cells.

\section{KCTD1 Enhances HUVEC Tube Formation and Matrigel Plug Angiogenesis with Increased M2 Macrophage Infiltration}

The single cell RNA sequencing data showed KCTD1 was expressed in endothelial cells of HCC (Additional file 6: Figure S4a). RNA sequencing analysis reveal that KCTD1 expression is positively correlated with vascular related gene expression (Fig. 4a). Moreover, KCTD1 influences the expression of the vascular marker CD31 and so whether KCTD1 modulated angiogenesis in HCC is studied. A stable HUVEC line that overexpressed KCTD1 protein is generated (Fig. 4b) and it is found that KCTD1 enhances proliferation and migration of these HUVECs in vitro (Fig. 4c-f, Figure S4b-d). KCTD1 overexpression induces HUVEC tube formation (Fig. $4 \mathrm{~g}$ ) and increases the branch counts of formed tubes and the number of HUVEC-formed tubes (Figure S4e). The supernatant from KCTD1-overexpressing Huh7 and HepG2 cells increases the number of HUVEC-formed tubes (Fig. 4h and Figure S4f-g), while the supernatant from KCTD1-knockdown MHCC97H cells decreases the number of HUVEC-formed tubes (Figure S4h). The effects of KCTD1 overexpression on new vessel formation in Matrigel plugs are investigated by subcutaneously injecting growth factor-reduced Matrigel with $800 \mathrm{ng} / \mathrm{ml} \mathrm{bFGF}$, HUVECs and Huh7 cells into the lower backs of 5-week-old female nude mice. The Matrigel plugs containing control cells are transparent indicating no new blood vessel formation, whereas the plugs containing KCTD1 cells are red and blood and blood vessels appear in two weeks (Fig. 4i). Correspondingly, KCTD1 overexpression induces phosphorylation of AKT, and VEGFR2 and promotes the expression of VEGF, HIF- 
1a and ARNT in HUVECs (Fig. 4j). These finding indicate that KCTD1 overexpression promotes the angiogenesis through activating the VEGF signaling cascade in vitro and in vivo.

Tumor-associated macrophages (TAMs) as a vital population in the tumor microenvironment (TME) are closely associated with tumor survival, angiogenesis, chemoresistance and immunosuppression, [20] and the correlation between the expression of CD31 and the M2 macrophage marker Arg1 in the HCC samples is analyzed. CD31 expression is upregulated in HCC tissues compared with healthy, adjacent liver tissues (Fig. 5a-b). The expression of CD31 is positively associated with KCTD1 expression in the HCC cases and high levels of CD31 are primarily observed from HCCs with high KCTD1 expression (Additional file 7: Table S3). The TCGA database indicates that KCTD1 is positively related with another vascular marker CD34 (Fig. 5c) and KCTD1 expression is positively associated with Arg1 expression in HCCs (Fig. 5d-e and Additional file 8: Table S4). The TIMER software shows that KCTD1 expression is positively correlated with the abundance of macrophage in HCCs (Fig. 5f) and the TCGA database reveals that KCTD1 expression is closely associated with the M2 macrophage in HCCs (Additional file 9: Figure S5A). By using qRT-PCR, lower levels of the M2 markersTGF- $\beta$, IL-10 and Arg1 are detected but higher levels of the M1 markersIL-1 $\beta /$ IL-12/TNFa/iNOS are observed from the KCTD1-knockdown HCC cells (Fig. $5 \mathrm{~g}$ ). The U937-differentiated M0, M1, M2 cells are cultured (Figure S5b) and M0 cells are cocultured with the supernatant from MHCC97H cells. The levels of M1-macrophage marker genes IL-12, IL-1 $\beta$, TNFa and iNOS are increased, while the level of M2-macrophage marker genes TGF- $\beta$, IL-10 and Arg1 is downregulated in the M0 cells cocultured with the supernatant from KCTD1-knockdown MHCC97H cells (Figure S5c). KCTD1 knockdown could induce M0 macrophage expressing M1 characteristic genes, and polarize M0 macrophages into M1 macrophages, indicating that KCTD1 expression is associated with the tumor microenvironment.

\section{KCTD1 Activates the HIF-1a/VEGF Pathway by Interacting with HIF-1a}

Hypoxia-inducible factor 1 alpha (HIF-1a) mediates the hypoxia-induced expression of VEGF, which plays a central role in angiogenesis and tumor progression [21]. Since KCTD1 has a key role in angiogenesis, whether KCTD1 regulates the HIF-1 a/VEGF pathway in HCC cells is investigated. KCTD1 overexpression promotes the expression of the p-ARK/HIF-1a-ARNT/VEGF cascade in Huh7 cells (Fig. 6a), while KCTD1 knockdown decreases the expression of these proteins in MHCC97H cells (Fig. 6b). Notably, KCTD1 affects the expression of GPC3 and AFP, which are HCC clinical biomarkers. Decreased levels of ARNT and HIF-1a are observed from the KCTD1 shRNA group according to the mouse subcutaneous tumor tissues (Additional file 10: Figure S6a). The KCTD1 protein stability assays are performed and as shown in Fig. 6c-d, more significant decrease in HIF-1a/ARNT proteins is induced in the KCTD1-knockdown $\mathrm{MHCC} 97 \mathrm{H}$ cells than in the control cells treated with different concentrations of cycloheximide $(\mathrm{CHX})$ at different time points. In contrast, an increasing amount of HIF-1 a proteins is measured from the KCTD1 overexpressing Huh7 cells treated with $\mathrm{CHX}$ (Figure S6b). $\mathrm{CoCl}_{2}$ induces the expression of HIF-1a in the HCC cells [22], KCTD1 overexpression leads to increased HIF-1 a proteins, but KCTD1 knockdown results in decreased HIF-1 a proteins (Figure S6c). These data strongly suggest that KCTD1 regulates the stability of HIF-1a/ARNT heterodimer proteins. 
To further disclose the regulatory relationship of KCTD1 and HIF-1a/ARNT genes, co-immunoprecipitation assays are carried out. Endogenous HIF-1a is detected from immune complexes of overexpressed KCTD1 (Fig. 6e) and endogenous ARNT cannot appear from the immunoprecipitates of overexpressed KCTD1 (Fig. 6f), suggesting an interaction between KCTD1 and HIF-1a. As reported, ARNT forms a heterodimer with HIF-1 a [23-25]. HIF-1a is found from immunoprecipitates of ARNT, while preimmune IgG could not precipitate any specific protein (Fig. $6 \mathrm{~g}$ ). Chromatin immunoprecipitation using anti-KCTD1 antibodies shows enrichment of VEGF promoter fragments with HIF-1a-binding sites, indicating that KCTD1 forms a complex with HIF-1a/ARNT and binds with the VEGF promoter via HIF-1a binding sites [26] (Fig. 6h). Taken together, these data show that KCTD1 is able to bind with the HIF-1a/ARNT heterodimer to regulate the VEGF signaling pathway.

\section{Sorafenib Downregulates KCTD1 Expression to Increase the Cytotoxicity of HCC Cells both in vitro and in vivo}

HCC has a poor prognosis due to its high incidence of recurrence and metastasis and postoperative chemotherapy is necessary because of high drug resistance [27]. The effects of KCTD1 knockdown on the chemosensitivity of HCC cells are studied. The MTT absorbance decreases by $27 \%$ in the KCTD1knockdown HCC cells compared to the control cells, while $69 \%$ reduction in the MTT absorbance appears from the KCTD1-knockdown HCC cells treated with sorafenib (Fig. 7A and Additional file 11: Figure S7a), suggesting that the combination of KCTD1 knockdown with sorafenib increases the HCC cell cytotoxicity.

After 24-h treatment with sorafenib, cell apoptosis of the KCTD1 shRNA-infected and parental MHCC97H cells is detected by flow cytometry. The rate of apoptosis increases for cells with sorafenib treatment and KCTD knockdown, as independently and synergistically compared to the proportion of the control cells (Fig. 7B and Figure S7B). Sorafenib markedly decreases the expression levels of KCTD1, HIF-1a, and ARNT proteins (Fig. 7c and Figure S7c). The effects of KCTD1 downregulation and sorafenib on tumor formation in subcutaneous and intrahepatic mouse models are probed. Both treatments synergistically decrease the size of subcutaneous tumors and 4 mice display complete tumor remission (Fig.7d and Figure S7d). Both treatments result in smaller tumor foci in the mouse livers than the control group and single treatment group (Fig. 7e and Figure S7e). Clinically, the low expression of KCTD1 leads to a prolonged median overall survival of $30.9 \%$ in 29 sorafenib-treated patients with recurrent HCC, although the sample number is so small that there is no statistical significance (Figure S7f). The results reveal that KCTD1 knockdown and sorafenib synergistically inhibit HCC progression.

\section{miR-129-5p Targets the KCTD1 3' UTR and Decreases the Oncogenic Effects of KCTD1 in HCC in vitro as well as in vivo}

Because KCTD1 downregulation suppresses HCC progression, efficient miRNAs are predicted by multiple algorithms (TargetScan, miRWalk, RNA22 and miRanda) to identify miRNAs targeting the KCTD1 3' UTR and three potential miR-129-5p binding sites are found (Fig. 8a). Wildtype or mutated KCTD1 3'-UTR luciferase reporter vectors are cotransfected into HEK293 cells with miR-129-5p mimics. The overexpression of miR-129-5p suppresses the luciferase activities of 3' UTR of the KCTD1 gene (Fig. 8b) 
and decreases the protein expression of KCTD1 (Fig. 8c). However, miR-129-5p showed no effect on the luciferase activity of mutated miR-129-5p MRE seed sequences of KCTD1 (Additional file 12: Figure S8a), indicating that the predicted three miR-129-5P binding sites mediate binding of miR-129-5p with KCTD1. These data indicate that miR-129-5p specifically targets the 3' UTR region of KCTD1 gene and downregulates KCTD1 expression.

To demonstrate the critical role of miR-129-5p in KCTD1-mediated HCC cell growth and angiogenesis, MHCC97H cells are infected with miR-129-5p LV (Figure S8b), miR-129-5p dramatically suppresses growth (Fig. 8d) and migration of the MHCC97H cells (Fig. 8e and Figure S8c). The supernatant from miR-129-5p-overexpressing MHCC97H cells suppresses significantly the tube-like structure formation of the HUVECs (Fig. $8 \mathrm{f}$ and Figure S8d). miR-129-5p is positively linked with overall survival time of patients with HCC according to TCGA database previously reported [28] (Figure S8e). The results indicate that the tumor inhibitor miR-129-5p decreases the tumor-promoting and vascularization effects of HCC.

Finally, mouse HCC models are adopted to analyze the combined effects of miR-129-5p and sorafenib. Firstly, miR-129-5p suppresses subcutaneous tumor formation in nude mice (Fig. $8 \mathrm{~g}$ and Figure S8f) and exosomes from the supernatant of miR-129-5p-infected MHCC97H cells are injected into the tail veins of an intrahepatic tumor model in nude mice leading to a smaller size of tumor foci in the livers. The combination of both exosomes and sorafenib treatment synergistically reduces the size of the tumor foci in liver tissues compared with those in the control groups (Fig. 8h and Figure S8g). The morphology of isolated exosomes is examined by TEM (Figure S8h) and expression of exosome markers CD81 and CD63 is confirmed (Figure S8i). Expression level of miR-129-5p is markedly increased in exosomes from the supernatant of miR-129-5p-LV infected MHCC97H cells (Figure S8J) and these results suggest that miR-129-5p combined with sorafenib can suppress intrahepatic tumor development in vivo.

\section{Discussion}

The biological importance of KCTD1 in transcription regulation, adipogenesis, and SEN disease has been studied $^{[11,12,15]}$, but the potential function of KCTD1 in malignant tumorigenesis remains unknown. The TCGA data show that KCTD1 is highly expressed in most tumor tissues and the HCCDB website shows that KCTD1 is significantly upregulated in HCC tumors. In the current study, the variable level of KCTD1 expression is found in HCC tissues, but high expression of KCTD1 is detected from $47 \%$ of HCC samples. KCTD1 expression is positively associated with tumor grade and moreover, over $70 \%$ of patients with increased KCTD1 expression show enhanced CD31 protein levels, while $54 \%$ of patients with decreased KCTD1 expression reveal lower CD31 levels (Table S3). The positive correlation between KCTD1 and CD31 is demonstrated in both HCC tissues and HCC cell lines. 54.5\% of patients with increased KCTD1 expression show upregulated Arg1 levels (Table S4). The cell-surface marker GPC3 is specifically expressed at high levels in HCCs and increased in HCC patient serum, and GPC3 also serves as a serum marker of HCC [29]. Serum alpha-fetoprotein (AFP) is used as a tumor marker for detection and diagnosis of HCC patients [30]. Overexpression of the KCTD1 protein increases the protein levels of AFP and GPC3 in HCC cell lines and simultaneous identification of KCTD1 and GPC3/AFP may 
improve the sensitivity for diagnosis of HCC. The data thus indicate the potential clinical importance of KCTD1 in molecular classification, clinical characteristics, and detection of HCC.

Our functional assays confirm that KCTD1 overexpression promotes the in vitro growth, migration, and invasion of HCC cells. These malignant features are ameliorated by KCTD1 knockdown or application of its upstream regulator miR-129-5p, which targets the KCTD1 3' UTR. miR-129-5p has been reported to block HCC progression [31]. The role of KCTD1 overexpression and knockdown in regulating HCC cell growth, migration, and invasion is further demonstrated by murine subcutaneous and pulmonary metastasis. KCTD1 significantly enhances the tumor formation ability and increases the number of lung tumor nodules in vivo, while KCTD1 knockdown suppresses these effects. As expected, miR-129-5p mimics downregulated KCTD1 expression and exerts tumor suppressive effects. Owing to the tumorpromoting role of KCTD1, whether KCTD1 is directly associated with oncogenesis. KCTD1 overexpression in nontransformed NIH3T3 mouse fibroblast cells is studied to examine the malignant transformation by cell growth and colony formation. KCTD1 overexpression results in increased cell proliferation, colony formation, and spindle morphology as a transformed phenotype. KCTD-overexpressing NIH3T3 cells form tumors within 6 weeks, while the control cells develop very small tumors indicating the oncogenic ability of the KCTD1 protein.

Tube formation of endothelial cells is an important step during neoangiogenesis leading to the formation of a complicated network of vessels and capillaries [4]. VEGF regulates tumor angiogenesis and stimulates vascular endothelial cell growth, survival, and proliferation [32]. Although some factors such as specific activator 1 (SP1), and HIF-1a have been found to regulate VEGF [33-35], whether KCTD1 participates in VEGF-related angiogenesis remains unclear. In this study, it is found that KCTD1 can enhance growth, migration, and tube formation of HUVECs and increase the number of vessels in 3D Matrigel plugs to enhance HCC angiogenesis. ARNT dimerizes with HIF-1a, promoting the expression of VEGF upon binding with the hypoxia-response element (HRE) in the VEGF promoter [36, 37]. The VEGF ligands such as VEGF, Ang1/2, and neuropillin and their receptors including VEGFR1/2 and Tie2 are critical regulators of angiogenesis and vasculogenesis pathways [38]. Mechanistically, KCTD1 upregulates the AKT/HIF-1a/VEGF pathway by interacting with HIF-1a. KCTD1 can bind to HIF-1a and enhance the stability of the HIF-1a/ARNT heterodimer, consequently increasing the expression of downstream VEGF and activating the VEGF/ p-VEGFR2/p-AKT/HIF-1a signaling cascade to form a positive feedback loop (Fig. 8I). The expression of CD31 is enhanced in KCTD1-overexpressing HCC samples and mouse tissues. The M2-polarized macrophages are linked with angiogenesis in human cancers [39]. Polarized macrophages are divided into either classically (M1) or alternatively (M2) activated macrophages [40]. The proinflammatory M1 macrophages release many oxidative metabolites and cytokines for host defense and tumoricidal activity whereas the anti-inflammatory M2 macrophages induced by IL4/IL13 promote tumor angiogenesis and tissue remodeling [41]. The M1/M2 ratio increases to reduce the immune suppressive function of myeloid cells and promote $T$ cell effector activation and CD8 ${ }^{+}$T-cell-mediated cytotoxicity [42]. Interestingly, KCTD1 promotes angiogenesis with increased M2 macrophage infiltration, concurrent with increased the M2 marker Arg1 in the HCC samples. 
The M0 cells of U937 macrophages are cocultured from the supernatant of HCC cells with KCTD1 knockdown and the expression of M1 marker genes is increased but that of M2 marker genes is suppressed in the induced M0 cells. All in all, the data show that KCTD1 is an important regulator of angiogenesis and tumor immunity.

Sorafenib suppresses the RAS/RAF/MEK/ERK pathway, and blocks tumor angiogenesis in human HCC [43]. Sorafenib significantly enhances KCTD1 knockdown-induced death of HCC cells and the synergistic function is demonstrated in subcutaneous and intrahepatic HCC mouse models, indicating that KCTD1 knockdown increases the sensitivity of HCC cells to sorafenib. Sorafenib significantly downregulates the KCTD1 protein in the HCC cell lines, suggesting that KCTD1 could be an important and novel target of sorafenib. More importantly, miR-129-5p combined with sorafenib synergistically suppresses development of HCC. Exosomes can serve as cargos to deliver miRNAs to target specific organs conveniently and efficiently $[44,45]$ and exosomes derived from the miR-129-5p-infected HCC cell line also significantly reduce $\mathrm{HCC}$ tumor size. These results suggest the possibility of a combined therapy consisting of sorafenib and KCTD1 knockdown for treating advanced HCC.

Principal transcription factors, CCAAT/enhancer-binding proteins (C/EBPa and $\beta)$ and the peroxisome proliferator activated receptor $\mathrm{Y}$ (PPARY) drive fat cell differentiation as early regulators of adipogenesis, whereas fatty acid-binding protein 4 (FABP4) and adiponectin are critical for the differentiation into mature adipocytes [46]. Genome-wide association studies (GWAS) have reported KCTD1, IGF1, GCKR, IRS1, GCK1 and PPARG as good candidates for insulin resistance and type 2 diabetes (T2D) [47]. KCTD1 promotes adipogenesis by removing the interacting partner AP-2a from the nucleus, while Kctd1-specific siRNA inhibits preadipocyte differentiation by reducing the expression of these specific adipogenic markers C/ebpa, Glut4, adiponectin and Ppary2 [11]. The insulin-regulated glucose transporter GLUT4 is critical for insulin-mediated glucose uptake in adipocytes and muscle cells [48]. KCTD15, a homolog of KCTD1 in Danio Rerio, interacts with adipogenesis GRP78 and is involved in food uptake and obesity control [49]. These results suggest the potential importance of KCTD1 in adipocyte metabolism. KCTD1 upregulates the levels of phosphorylated AKT in HCC cells while AKT triggers hepatic lipogenesis, hepatic steatosis, and activation of mTORC1 signaling in HCC [50]. KCTD1 may enhance lipogenesis, hepatic steatosis, and HCC. It is interesting to elucidate the significance and cross-interactions of KCTD1 among hepatic metabolism, tumor immunity, and sorafenib resistance to further expand our knowledge of KCTD1 and reveal the therapeutic implications of KCTD1 in individual HCC cases.

\section{Conclusions}

In summary, our study suggests that KCTD1 promotes proliferation, migration, and angiogenesis of HCCs by activating the HIF-1a/VEGF pathway. KCTD1 knockdown increases the cytotoxicity of the angiogenesis inhibitor sorafenib in HCCs and KCTD1 exerts an oncogenic role in development and progression of HCC. KCTD1 upregulation may serve as a marker in diagnosis as well as a target for therapy in high-grade HCC. Understanding functions and mechanisms of KCTD1 leading to HCC is crucial to future intervention and inhibition of angiogenesis in HCC. 


\section{List Of Abbreviations}

AFP: alpha fetoprotein; APC: adenomatous polyposis coli; ARNT: Arylhydrocarbon Receptor Nuclear Translocator; BOFS: Branchio-Oculo-Facial syndrome; CDX: cell-line-derived xenograft; C/EBPs: CCAAT/enhancer binding proteins; ChIP: chromatin immunoprecipitation; CHX: cycloheximide; CK1: casein kinase 1; $\mathrm{CM}$ : conditioned medium; $\mathrm{CoCl}_{2}$ : cobalt chloride; COIP: co-immunoprecipitation; DMSO: dimethylsulfoxide; FABP4: fatty acid-binding protein 4; FAS: fatty acid synthase; GSK-3ß: glycogen synthase kinase-3ß; GWAS: Genome-wide association studies; HCC: hepatocellular cancer; HE: Hematoxylin and Eosin; HIF-1a: hypoxia inducible factor-1; HRE: hypoxia-response element; HUVEC: Human Umbilical Vein Endothelial Cells; IHC: immunohistochemical; KCTD1: Potassium channel tetramerization domain-containing 1; LV: lentivirus; M1: classically activated macrophages; M2: alternatively activated macrophages; MOI: multiply of infection; MRE: microRNA response element; MTT: 3-(4,5-dimethylthiazol-2-yl)-2,5-diphenytetrazolium bromide; NC: negative control; PET: polyethylene terephthalate; PI: propidium iodide; PPARY: peroxisome proliferator-activated receptor $\gamma$; s.c.: subcutaneous; SEN: Scalp-Ear-Nipple syndrome; SP1: specific activator 1; STAT3: signal transducers and activators of transcription 3; T2D: type 2 diabetes; TAMs: Tumor-associated macrophages; TCGA: the Cancer Genome Atlas; TEM: Transmission electron microscope; TISCH: Tumor Immune Single-cell Hub; TME: tumor microenvironment; VEGF: vascular endothelial growth factor; VEGFR2: VEGF receptor 2; UTR: $3^{\prime}$ untranslated region

\section{Declarations}

\section{Ethics approval and consent to participate}

This study was approved by the Ethics Committee of Hunan Normal University.

\section{Consent for publication}

All authors reviewed and approved the manuscript.

\section{Availability of data and material}

All data generated or analyzed during this study are included in this published article and its supplementary files.

\section{Competing interests}

The authors declare no competing interests.

\section{Funding}

This work was supported by the National Natural Science Foundation of China (No. 81272190), Key grant of research and development in Hunan Province (No. 2020DK2002), Key project of Hunan Provincial 
Education Department (No. 19A310), Cooperative Innovation Center of Engineering and New Products for Developmental Biology of Hunan Province (No. 20134486), as well as City University of Hong Kong Strategic Research Grant (No. 7005264).

\section{Authors' contributions}

$X D$ conceived and designed this study. XZ, ZL, LL, LC, CX and MO carried out the experiments. LL, HZ, KX, $L Y, W H$ and $X D$ performed the statistical analysis and analyzed the data. $X D, C Z$ and $P C$ supervised the study. XD drafted the manuscript. All authors read and approved the final manuscript.

\section{Acknowledgements}

The authors would like to thank Jianlin Zhou, professor, College of Life Science, Hunan Normal University, for providing some reagents and advice.

\section{Author details}

1 The National \& Local Joint Engineering Laboratory of Animal Peptide Drug Development, College of Life Science, Hunan Normal University, Changsha, 410081, China. ${ }^{2}$

Key Laboratory of Model Animals and Stem Cell Biology in Hunan Province, School of Medicine, Hunan Normal University, Changsha, 410013 , China. ${ }^{3}$ College of Engineering and Design, Hunan Normal University, Changsha, 410081 , China. ${ }^{4}$ Department of Neurosurgery, Xiangya Hospital of Central South University, Changsha, Hunan, 410008 , China. ${ }^{5}$ Department of Hepatobiliary and Pancreatic Surgery, Xiangya Hospital of Central South University, Changsha, Hunan, 410008, China. ${ }^{6}$ Department of Physics, Department of Materials Science and Engineering, and Department of Biomedical Engineering, City University of Hong Kong, Tat Chee Avenue, Kowloon, Hong Kong, China.

\section{References}

1. Muto J, Shirabe K, Sugimachi K, Maehara Y. Review of angiogenesis in hepatocellular carcinoma. Hepatol Res. 2015; 45:1-9.

2. Ribatti D, Vacca A, Nico B, Sansonno D, Dammacco F. Angiogenesis and anti-angiogenesis in hepatocellular carcinoma. Cancer Treat Rev. 2006; 32:437-44.

3. Nilsson M, Heymach JV. Vascular endothelial growth factor (VEGF) pathway. J Thorac Oncol. 2006; 1:768-70.

4. Ferrara N, Gerber HP, LeCouter J. The biology of VEGF and its receptors. Nat Med. 2003; 9:669-76.

5. Lee JE, Kim C, Yang H, Park I, Oh N, Hua S, Jeong H, An HJ, Kim SC, Lee GM, Koh GY, Kim HM. Novel glycosylated VEGF decoy receptor fusion protein, VEGF-Grab, efficiently suppresses tumor angiogenesis and progression. Mol Cancer Ther. 2015; 14:470-9.

6. Shibuya M, Claesson-Welsh L. Signal transduction by VEGF receptors in regulation of angiogenesis and lymphangiogenesis. Exp Cell Res. 2006; 312:549-60. 
7. Llovet JM, Ricci S, Mazzaferro V, Hilgard P, Gane E, Blanc JF, de Oliveira AC, Santoro A, Raoul JL, Forner A, Schwartz M, Porta C, Zeuzem S, Bolondi L, Greten TF, Galle PR, Seitz JF, Borbath I, Haussinger D, Giannaris T, Shan M, Moscovici M, Voliotis D, Bruix J, Group SIS. Sorafenib in advanced hepatocellular carcinoma. N Engl J Med. 2008; 359:378-90.

8. Ding XF, Luo C, Ren KQ, Zhang J, Zhou JL, Hu X, Liu RS, Wang Y, Gao X, Zhang J. Characterization and expression of a human KCTD1 gene containing the BTB domain, which mediates transcriptional repression and homomeric interactions. DNA Cell Biol. 2008; 27:257-65.

9. Ding X, Luo C, Zhou J, Zhong Y, Hu X, Zhou F, Ren K, Gan L, He A, Zhu J, Gao X, Zhang J. The interaction of KCTD1 with transcription factor AP-2alpha inhibits its transactivation. J Cell Biochem. 2009; 106:285-95.

10. Li X, Chen C, Wang F, Huang W, Liang Z, Xiao Y, Wei K, Wan Z, Hu X, Xiang S, Ding X, Zhang J. KCTD1 suppresses canonical Wnt signaling pathway by enhancing beta-catenin degradation. PLoS One. 2014; 9:e94343.

11. Pirone L, Smaldone G, Spinelli R, Barberisi M, Beguinot F, Vitagliano L, Miele C, Di Gaetano S, Raciti GA, Pedone E. KCTD1: A novel modulator of adipogenesis through the interaction with the transcription factor AP2alpha. Biochim Biophys Acta Mol Cell Biol Lipids. 2019; 1864:158514.

12. Marneros AG. AP-2beta/KCTD1 Control Distal Nephron Differentiation and Protect against Renal Fibrosis. Dev Cell. 2020; 54:348-66.e5.

13. Milunsky JM, Maher TA, Zhao G, Roberts AE, Stalker HJ, Zori RT, Burch MN, Clemens M, Mulliken JB, Smith $\mathrm{R}$, Lin AE. TFAP2A mutations result in branchio-oculo-facial syndrome. Am J Hum Genet. 2008; 82:1171-7.

14. Zhao F, Weismann CG, Satoda M, Pierpont ME, Sweeney E, Thompson EM, Gelb BD. Novel TFAP2B Mutations That Cause Char Syndrome Provide a Genotype-Phenotype Correlation. American Journal of Human Genetics. 2001; 69:695-703.

15. Marneros AG, Beck AE, Turner EH, McMillin MJ, Edwards MJ, Field M, de Macena Sobreira NL, Perez AB, Fortes JA, Lampe AK, Giovannucci Uzielli ML, Gordon CT, Plessis G, Le Merrer M, Amiel J, Reichenberger E, Shively KM, Cerrato F, Labow BI, Tabor HK, Smith JD, Shendure J, Nickerson DA, Bamshad MJ, University of Washington Center for Mendelian G. Mutations in KCTD1 cause scalpear-nipple syndrome. Am J Hum Genet. 2013; 92:621-6.

16. Smaldone G, Balasco N, Pirone L, Caruso D, Di Gaetano S, Pedone EM, Vitagliano L. Molecular basis of the scalp-ear-nipple syndrome unraveled by the characterization of disease-causing KCTD1 mutants. Sci Rep. 2019; 9:10519.

17. Hu L, Chen L, Yang L, Ye Z, Huang W, Li X, Liu Q, Qiu J, Ding X. KCTD1 mutants in scalp-ear-nipple syndrome and AP-2a P59A in Char syndrome reciprocally abrogate their interactions, but can regulate Wnt/ $\beta$-catenin signaling. Molecular Medicine Reports. 2020; 22:3895-903.

18. Hanahan D, Weinberg RA. The hallmarks of cancer. Cell. 2000; 100:57-70.

19. Chen C, Liang Z, Huang W, Li X, Zhou F, Hu X, Han M, Ding X, Xiang S. Eps8 regulates cellular proliferation and migration of breast cancer. Int J Oncol. 2015; 46:205-14. 
20. Ngambenjawong C, Gustafson HH, Pun SH. Progress in tumor-associated macrophage (TAM)targeted therapeutics. Adv Drug Deliv Rev. 2017; 114:206-21.

21. Gabrilovich DI, Ostrand-Rosenberg S, Bronte V. Coordinated regulation of myeloid cells by tumours. Nat Rev Immunol. 2012; 12:253-68.

22. Piret JP, Mottet $D$, Raes M, Michiels $C$. CoCl2, a chemical inducer of hypoxia-inducible factor- 1 , and hypoxia reduce apoptotic cell death in hepatoma cell line HepG2. Ann N Y Acad Sci. 2002; 973:443-7.

23. Wang GL, Jiang BH, Rue EA, Semenza GL. Hypoxia-inducible factor 1 is a basic-helix-loop-helix-PAS heterodimer regulated by cellular 02 tension. Proceedings of the National Academy of Sciences of the United States of America. 1995; 92:5510-4.

24. Ivan M, Kondo K, Yang H, Kim W, Valiando J, Ohh M, Salic A, Asara JM, Lane WS, Kaelin WG, Jr. HIFalpha targeted for VHL-mediated destruction by proline hydroxylation: implications for $\mathrm{O} 2$ sensing. Science. 2001; 292:464-8.

25. Jaakkola P, Mole DR, Tian YM, Wilson MI, Gielbert J, Gaskell SJ, von Kriegsheim A, Hebestreit HF, Mukherji M, Schofield CJ, Maxwell PH, Pugh CW, Ratcliffe PJ. Targeting of HIF-alpha to the von Hippel-Lindau ubiquitylation complex by 02-regulated prolyl hydroxylation. Science. 2001; 292:46872.

26. Sun Q, Zhou H, Binmadi NO, Basile JR. Hypoxia-inducible factor-1-mediated regulation of semaphorin 4D affects tumor growth and vascularity. J Biol Chem. 2009; 284:32066-74.

27. Zhang YS, Yuan FJ, Jia GF, Zhang JF, Hu LY, Huang L, Wang J, Dai ZQ. CIK cells from patients with HCC possess strong cytotoxicity to multidrug-resistant cell line Bel-7402/R. World J Gastroenterol. 2005; 11:3339-45.

28. Li S, Li J, Fei BY, Shao D, Pan Y, Mo ZH, Sun BZ, Zhang D, Zheng X, Zhang M, Zhang XW, Chen L. MiR27a promotes hepatocellular carcinoma cell proliferation through suppression of its target gene peroxisome proliferator-activated receptor gamma. Chin Med J (Engl). 2015; 128:941-7.

29. Zhu ZW, Friess H, Wang L, Abou-Shady M, Zimmermann A, Lander AD, Korc M, Kleeff J, Buchler MW. Enhanced glypican-3 expression differentiates the majority of hepatocellular carcinomas from benign hepatic disorders. Gut. 2001; 48:558-64.

30. Zhou L, Liu J, Luo F. Serum tumor markers for detection of hepatocellular carcinoma. World J Gastroenterol. 2006; 12:1175-81.

31. Han H, Li W, Shen H, Zhang J, Zhu Y, Li Y. microRNA-129-5p, a c-Myc negative target, affects hepatocellular carcinoma progression by blocking the Warburg effect. J Mol Cell Biol. 2016; 8:40010.

32. Breier G. Functions of the VEGF/VEGF receptor system in the vascular system. Semin Thromb Hemost. 2000; 26:553-9.

33. Pore N, Liu S, Shu HK, Li B, Haas-Kogan D, Stokoe D, Milanini-Mongiat J, Pages G, O'Rourke DM, Bernhard E, Maity A. Sp1 is involved in Akt-mediated induction of VEGF expression through an HIF-1independent mechanism. Mol Biol Cell. 2004; 15:4841-53. 
34. Forsythe JA, Jiang BH, lyer NV, Agani F, Leung SW, Koos RD, Semenza GL. Activation of vascular endothelial growth factor gene transcription by hypoxia-inducible factor 1. Mol Cell Biol. 1996; 16:4604-13.

35. Wei D, Le X, Zheng L, Wang L, Frey JA, Gao AC, Peng Z, Huang S, Xiong HQ, Abbruzzese JL, Xie K. Stat3 activation regulates the expression of vascular endothelial growth factor and human pancreatic cancer angiogenesis and metastasis. Oncogene. 2003; 22:319-29.

36. Semenza GL, Roth PH, Fang HM, Wang GL. Transcriptional regulation of genes encoding glycolytic enzymes by hypoxia-inducible factor 1 . J Biol Chem. 1994; 269:23757-63.

37. Jiang BH, Rue E, Wang GL, Roe R, Semenza GL. Dimerization, DNA binding, and transactivation properties of hypoxia-inducible factor 1. J Biol Chem. 1996; 271:17771-8.

38. Saharinen P, Eklund L, Pulkki K, Bono P, Alitalo K. VEGF and angiopoietin signaling in tumor angiogenesis and metastasis. Trends Mol Med. 2011; 17:347-62.

39. Mantovani A. La mala educacion of tumor-associated macrophages: Diverse pathways and new players. Cancer Cell. 2010; 17:111-2.

40. Martinez FO, Sica A, Mantovani A, Locati M. Macrophage activation and polarization. Front Biosci. 2008; 13:453-61.

41. Patel U, Rajasingh S, Samanta S, Cao T, Dawn B, Rajasingh J. Macrophage polarization in response to epigenetic modifiers during infection and inflammation. Drug Discov Today. 2017; 22:186-93.

42. De Henau O, Rausch M, Winkler D, Campesato LF, Liu C, Cymerman DH, Budhu S, Ghosh A, Pink M, Tchaicha J, Douglas M, Tibbitts T, Sharma S, Proctor J, Kosmider N, White K, Stern H, Soglia J, Adams J, Palombella VJ, McGovern K, Kutok JL, Wolchok JD, Merghoub T. Overcoming resistance to checkpoint blockade therapy by targeting PI3Kgamma in myeloid cells. Nature. 2016; 539:443-7.

43. Liu L, Cao Y, Chen C, Zhang X, McNabola A, Wilkie D, Wilhelm S, Lynch M, Carter C. Sorafenib blocks the RAF/MEK/ERK pathway, inhibits tumor angiogenesis, and induces tumor cell apoptosis in hepatocellular carcinoma model PLC/PRF/5. Cancer Res. 2006; 66:11851-8.

44. Zhang D, Lee H, Zhu Z, Minhas JK, Jin Y. Enrichment of selective miRNAs in exosomes and delivery of exosomal miRNAs in vitro and in vivo. Am J Physiol Lung Cell Mol Physiol. 2017; 312:L110-L21.

45. Zhai M, Zhu Y, Yang M, Mao C. Human Mesenchymal Stem Cell Derived Exosomes Enhance Cell-Free Bone Regeneration by Altering Their miRNAs Profiles. Adv Sci (Weinh). 2020; 7:2001334.

46. Moseti D, Regassa A, Kim WK. Molecular Regulation of Adipogenesis and Potential Anti-Adipogenic Bioactive Molecules. Int J Mol Sci. 2016; 17.

47. Chirita-Emandi A, Munteanu D, Andreescu N, Tutac P, Paul C, Velea IP, Pusztai AM, Hlistun V, Boiciuc C, Sacara V, Vudu L, Usurelu N, Puiu M. No clinical utility of common polymorphisms in IGF1, IRS1, GCKR, PPARG, GCK1 and KCTD1 genes previously associated with insulin resistance in overweight children from Romania and Moldova. J Pediatr Endocrinol Metab. 2019; 32:33-9.

48. Huang S, Czech MP. The GLUT4 glucose transporter. Cell Metab. 2007; 5:237-52. 
49. Smaldone G, Pirone L, Capolupo A, Vitagliano L, Monti MC, Di Gaetano S, Pedone E. The essential player in adipogenesis GRP78 is a novel KCTD15 interactor. Int J Biol Macromol. 2018; 115:469-75.

50. Zhang C, Hu J, Sheng L, Yuan M, Wu Y, Chen L, Wang G, Qiu Z. Ellagic acid ameliorates AKT-driven hepatic steatosis in mice by suppressing de novo lipogenesis via the AKT/SREBP-1/FASN pathway. Food Funct. 2019; 10:3410-20.

\section{Figures}




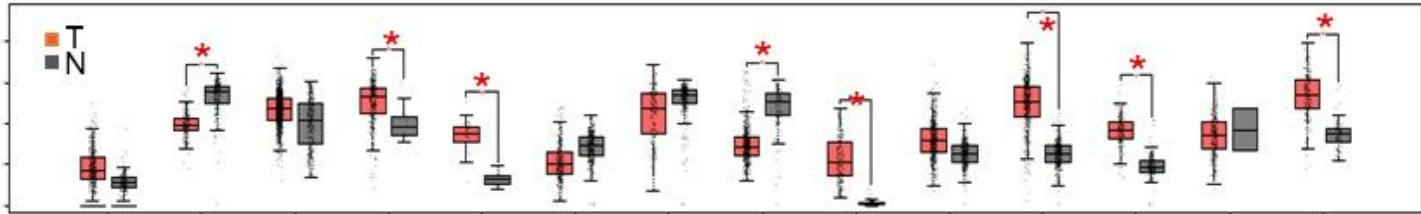

B

LIHC GBM BRCA CESC CHOL COAD ESCA KIRC LAML LUAD LUSC PAAD SARC U'CEC

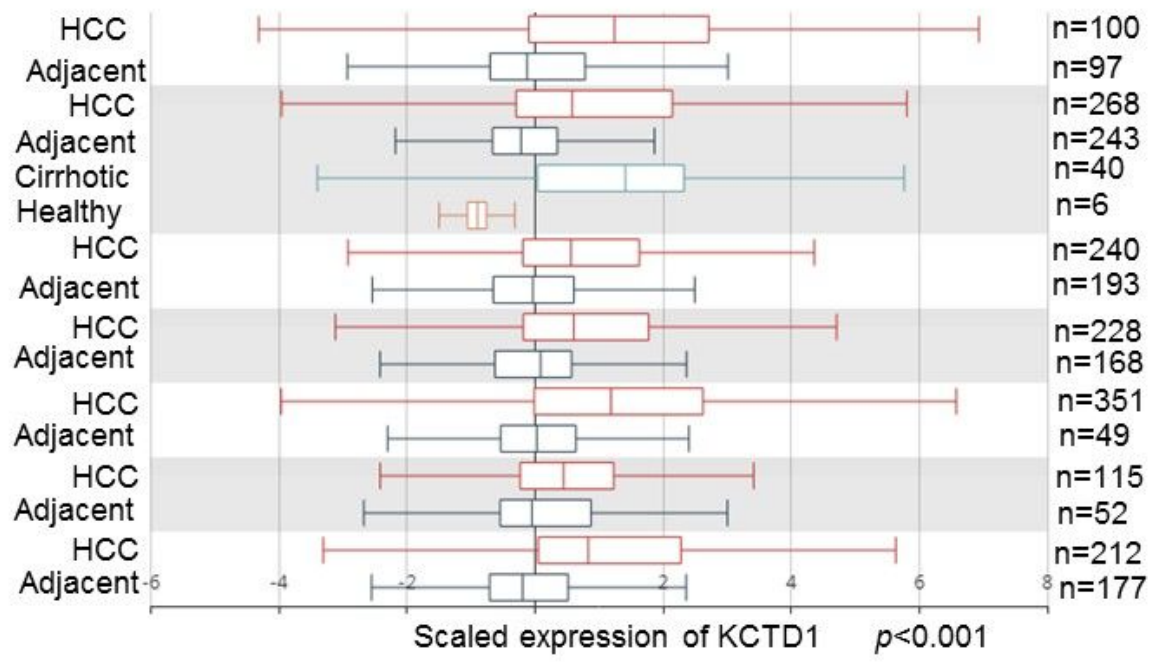

C
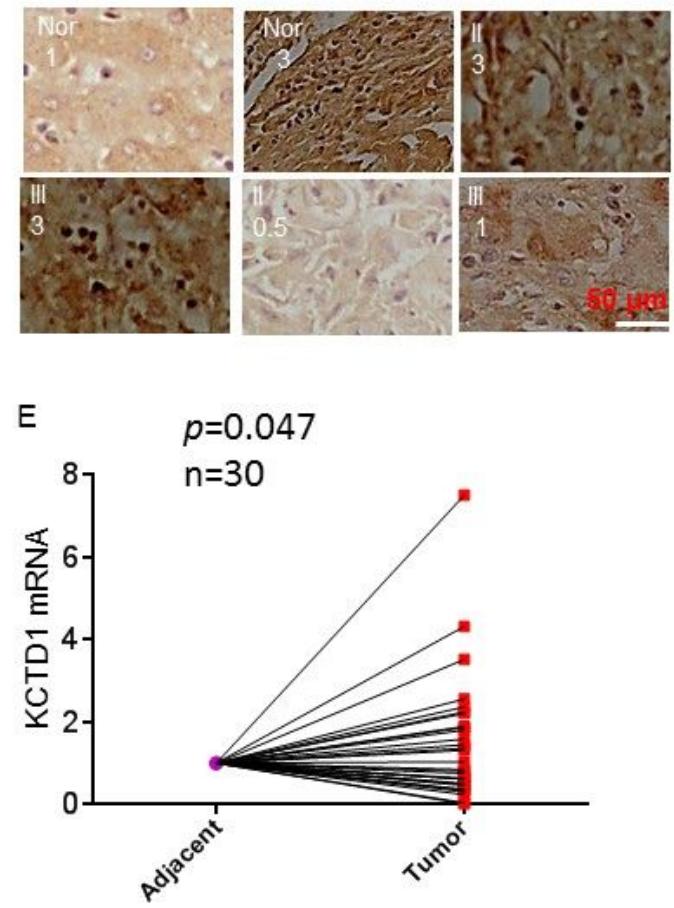
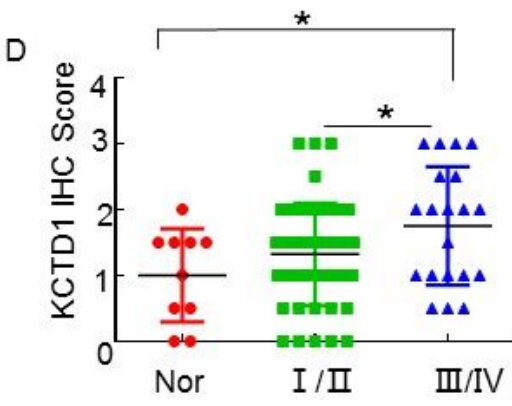

$\mathrm{F}$

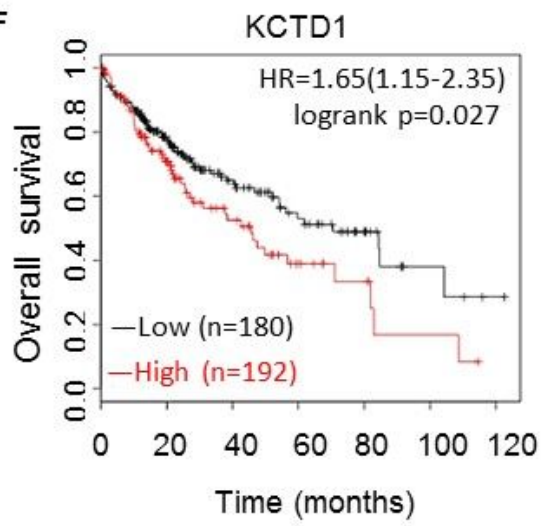

\section{Figure 1}

Expression levels of KCTD1 in both HCC tissues and HCC cell lines. a KCTD1 expression analyzed in the Cancer Genome Atlas (TCGA) database. b KCTD1 expression analyzed in the HCCDB database. c KCTD1 expression detected by IHC staining in $70 \mathrm{HCC}$ and 10 normal liver tissues. Nucleic expression of KCTD1 is shown brown in HCCs whereas the nucleus is stained blue. $\mathrm{d} \mathrm{IHC} \mathrm{score} \mathrm{of} \mathrm{HCCs} \mathrm{and} \mathrm{adjacent} \mathrm{normal}$ liver tissues detected with the polyclonal anti-KCTD1 antibodies. The staining intensities are scored as 0- 
3 and each symbol indicates an individual case. Statistical comparison between HCCs and non-tumor tissues is carried out by the SPSS software. *, $p<0.05$; $*$, $p<0.01$; e KCTD1 mRNA levels in paired hepatocellular cancer tissue samples detected by qRT-PCR; $f$ Correlation of KCTD1 expression and the overall survival of HCC patients in the TCGA cohort.

A

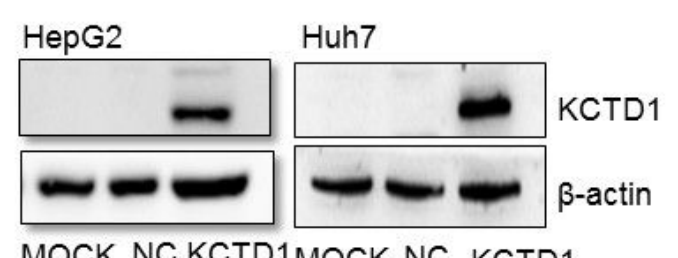

B
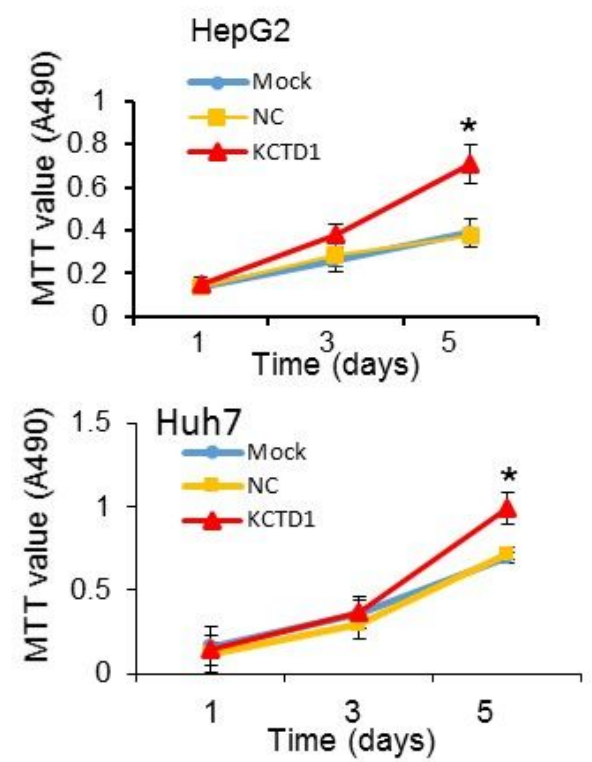

$\mathrm{F}$

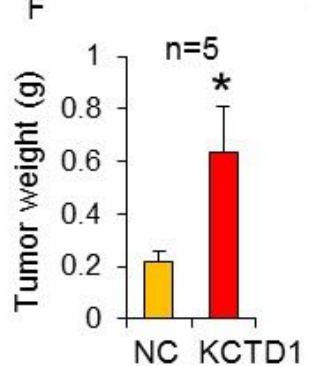

G
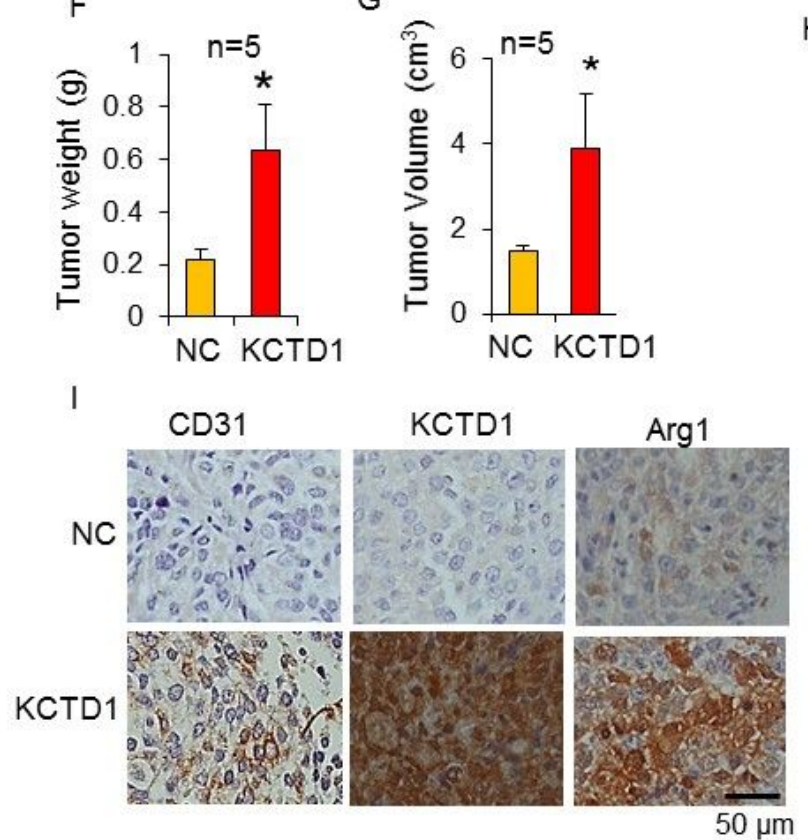

C
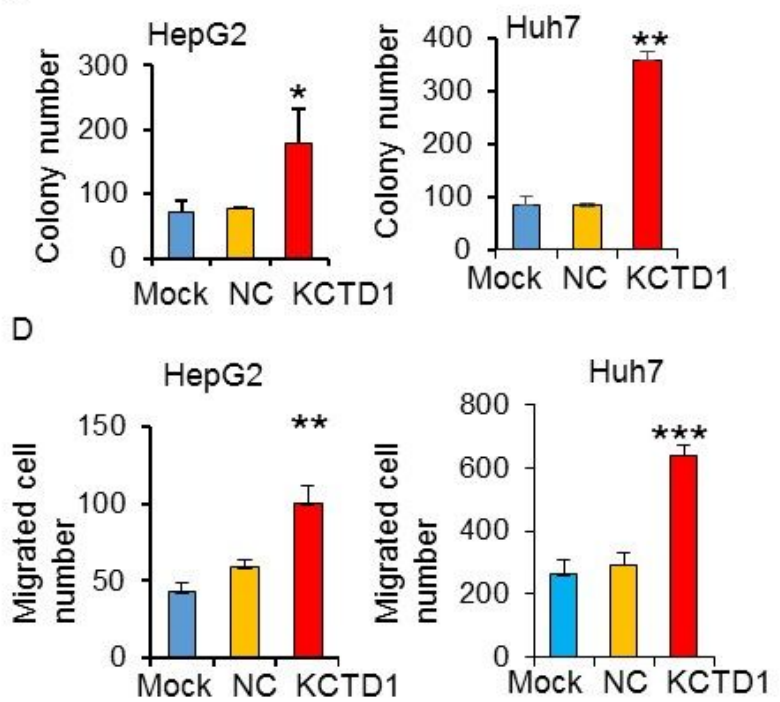

$E$
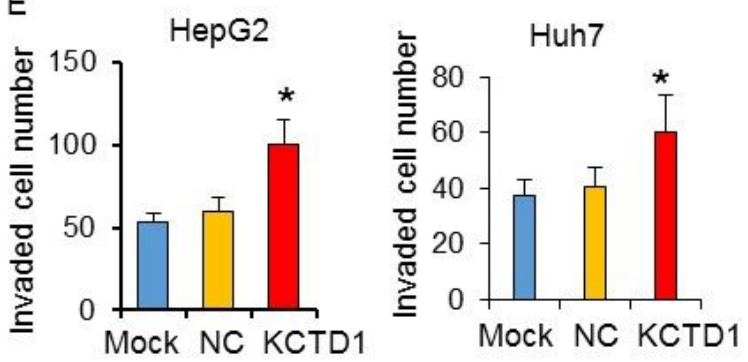

$\mathrm{H}$

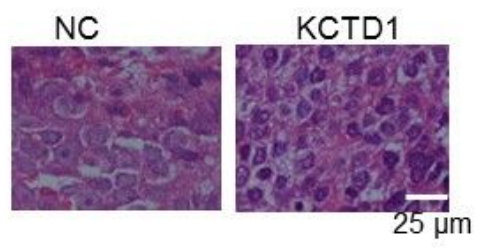

J
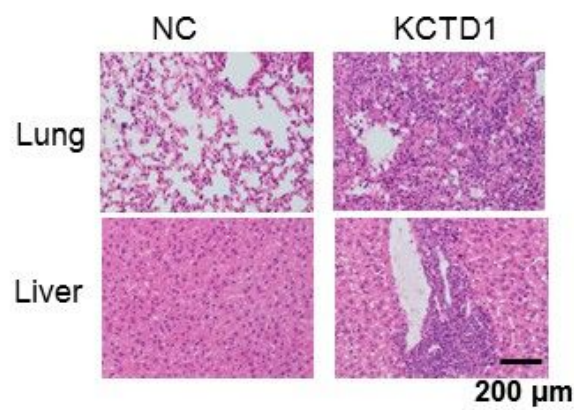

Figure 2 
Influences of KCTD1 overexpression on the in vitro and in vivo growth and migration of HCC cells. a Western blotting of KCTD1 expression in HCC cell lines detected with anti-KCTD1 antibodies; $b$ MTT analysis of both HCC cell lines infected with KCTD1 -LV. 3,000 cells are grown on a 96-well plate and cultured in complete DMEM medium. The absorbance at OD $490 \mathrm{~nm}$ is measured for $1-5$ days. c Liquid colony formation assay of both HCC cell lines infected with KCTD1-LV. 1,000 cells are plated on 6-well plates and cultured for 14 days. These cell colonies are fixed with absolute methanol, stained with Giemsa solution for counting. d Effects of KCTD1 overexpression on HCC cell migration using transwell migration assays. The average number of migrated HCC cells across the $8-\mu m$ pore-size PET membrane is shown. e Effects of KCTD1 overexpression on HCC cell invasion using Transwell Matrigel invasion assay. The average number of invaded HCC cells is shown. f Approximately $1 \times 107$ uninfected or infected Huh7 cells injected subcutaneously into the two points of the back of immunodeficient nude mice (female, $n=5 /$ group). After 4 weeks, the subcutaneous tumors are removed and weighed ( $f$ and $g$ ). The mean tumor weight/volume is obtained in triplicate. $\mathrm{h}$ HE staining carried out on serial $5 \mu \mathrm{m}$ paraffin sections of subcutaneous tumors derived from Huh7 cells. i IHC staining of CD31 and Arg1 expression in Huh7 tumors; $j$ Representative HE staining carried out on serial $5-\mu \mathrm{m}$ sections of mouse lung and liver tissues six weeks after tail-vein injection with Huh7 cells. These data indicate 3 independent experiments performed. $*, p<0.05, * \star, p<0.01$, and $* * *, p<0.001$. 

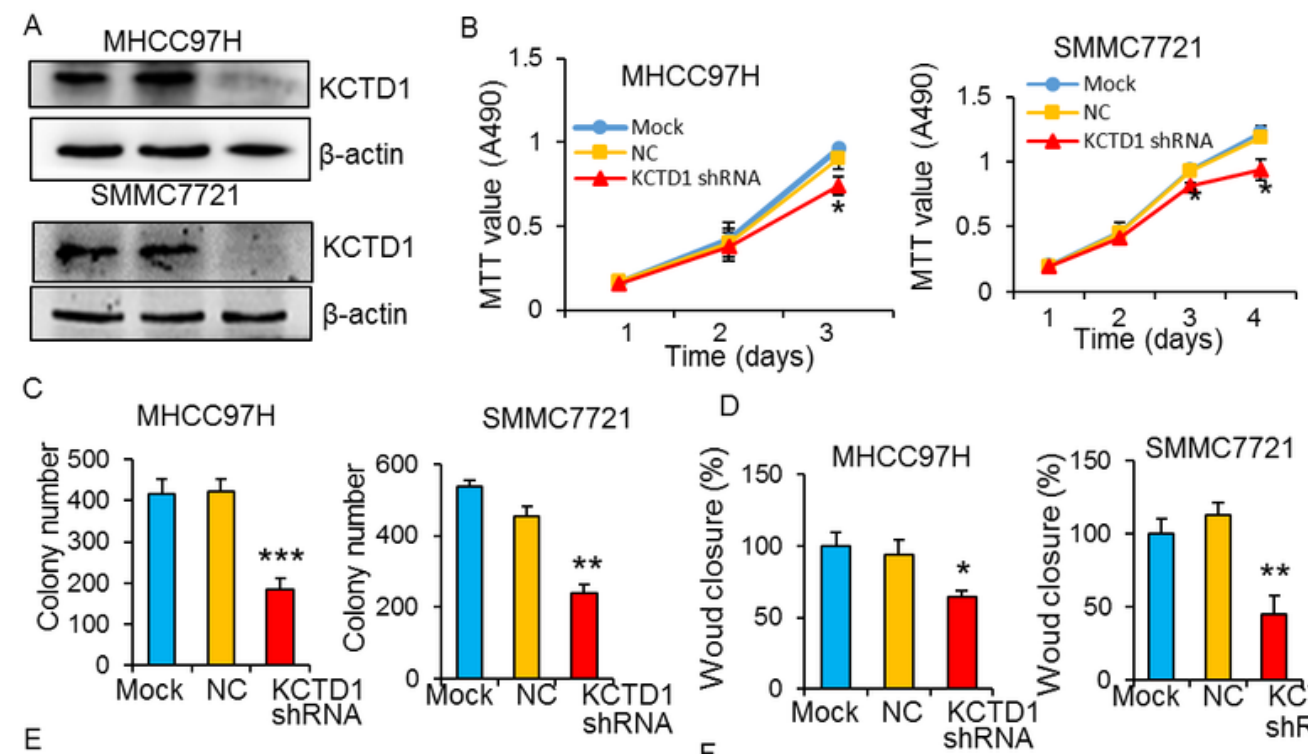

E
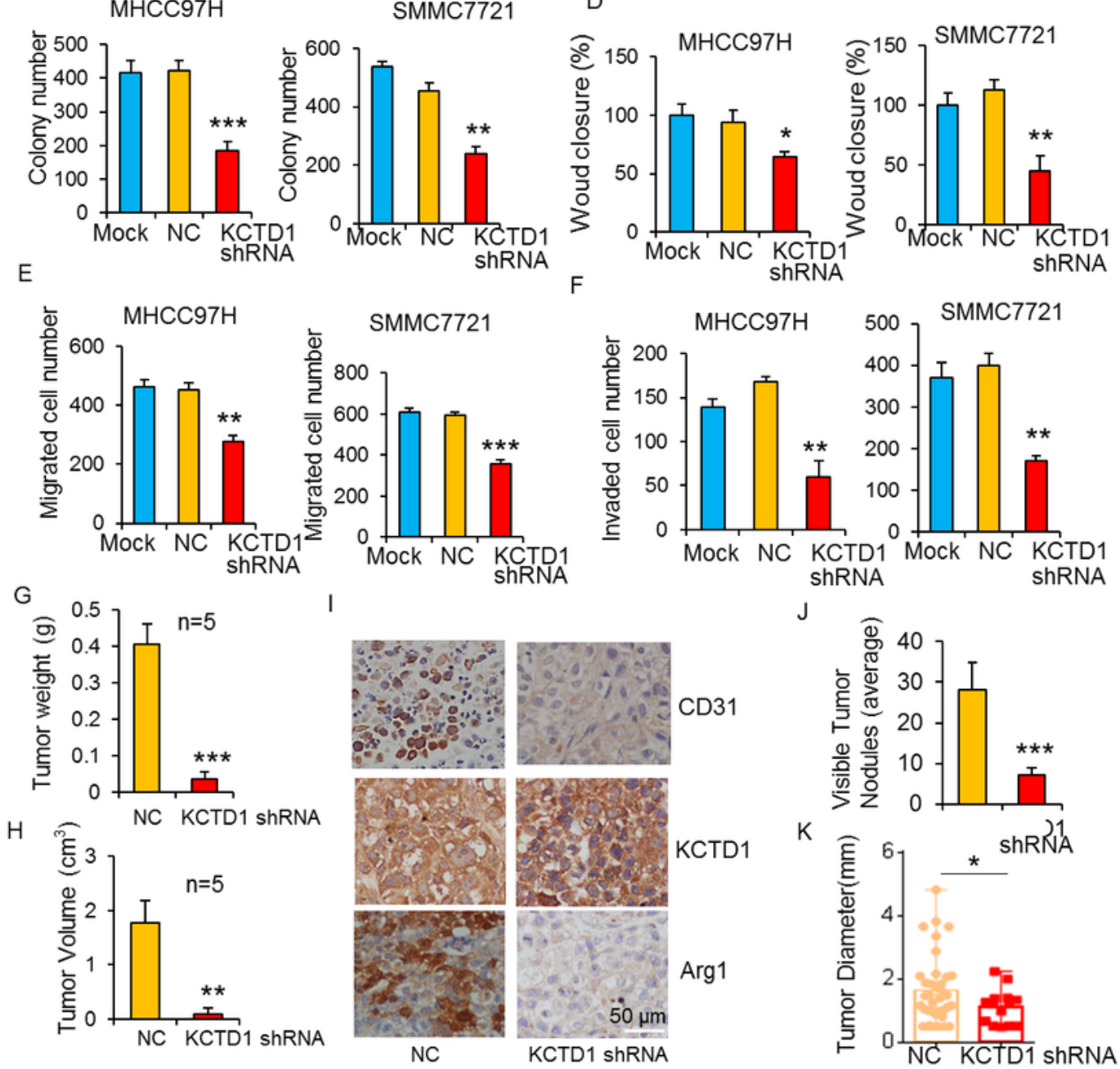

$\mathrm{H}$
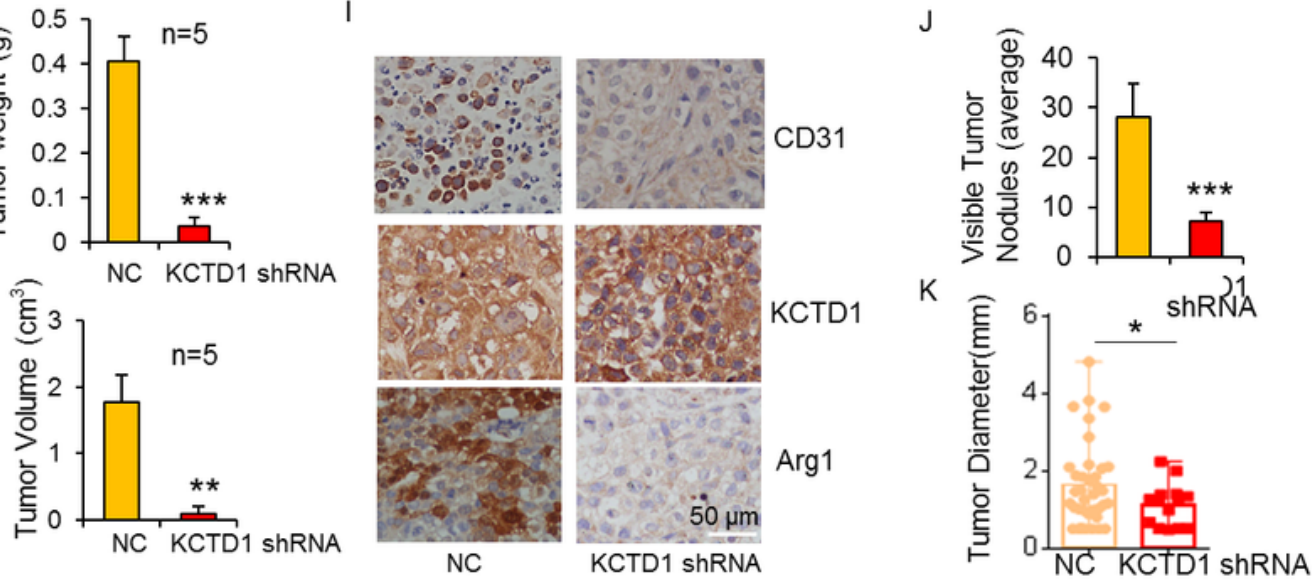

L

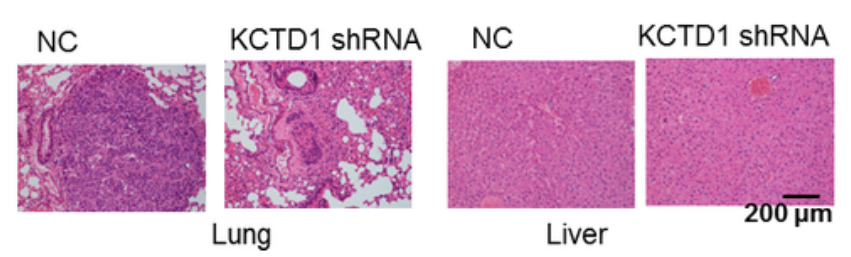

\section{Figure 3}

Effects of KCTD1 knockdown on HCC growth and migration in vitro. a Western blotting of NC shRNA and KCTD1 shRNA expression in MHCC97H and SMMC7721 cells detected with anti-KCTD1 antibodies. b MTT analysis of HCC cells infected with KCTD1 shRNA-LV. 2,000 cells are grown on a 96-well plate and cultured in complete DMEM medium. The absorbance is measured at the indicated time; c Liquid colony formation assay of HCC cells infected with KCTD1 shRNA-LV. 500 cells are plated on 6-well plates and 
cultured for 11 days. The cell colonies are fixed with absolute methanol, stained with Giemsa solution for counting. $d$ Scratch wound-healing assays carried out at the defined time in HCC cells infected with KCTD1 shRNA-LV. The areas are measured using the ImageJ software. e Effects of KCTD1 knockdown on transwell migration of $\mathrm{HCC}$ cells. The average number of migrated $\mathrm{HCC}$ cells through the $8-\mu \mathrm{m}$ pore-size PET membrane is shown. $f$ Effects of KCTD1 knockdown on HCC cell invasion through Transwell Matrigel invasion assay. The average number of invaded HCC cells is shown; $g$ About $8 \times 106$ infected MHCC97H cells injected subcutaneously into the two points of the back of nude mice (4-week old, female, $n=5$ /group). After 15 days, the tumors are removed, weighed and photographed. The mean weight $(\mathrm{g})$ and volume (h) of the subcutaneous tumors are obtained in triplicate. i IHC staining of CD31 and Arg1 expression in $\mathrm{MHCC} 97 \mathrm{H}$ tumors. The average number $(\mathrm{j})$ and diameter $(\mathrm{k})$ of lung nodules from nude mice were quantified six weeks after intravenous injection of MHCC97H cells ( $n=5$ per group). I HE staining carried out on serial $5-\mu \mathrm{m}$ sections of the lung and liver tissues after tail-vein injection with MHCC97H cells. These results were performed in triplicate. ${ }^{*}, p<0.05, * *, p<0.01$, and ${ }^{\star * \star}, p<0.001$. 
A

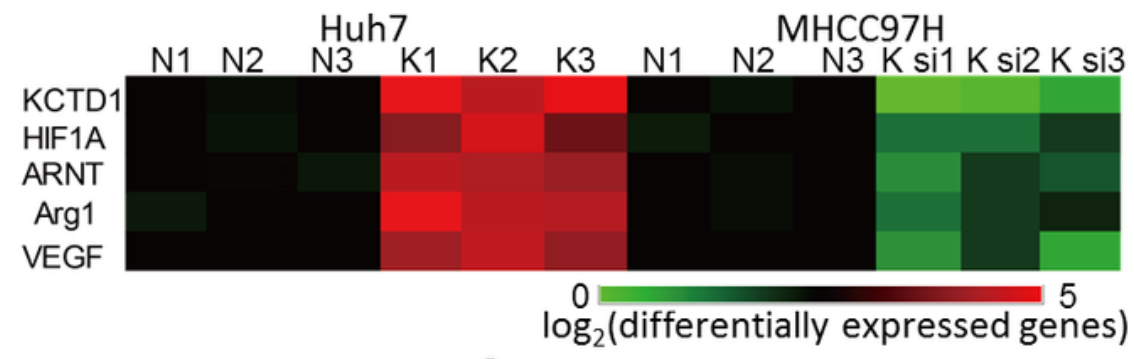

B

HUVEC

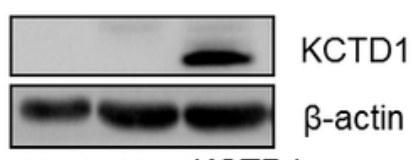

Mock NC KCTD1
C

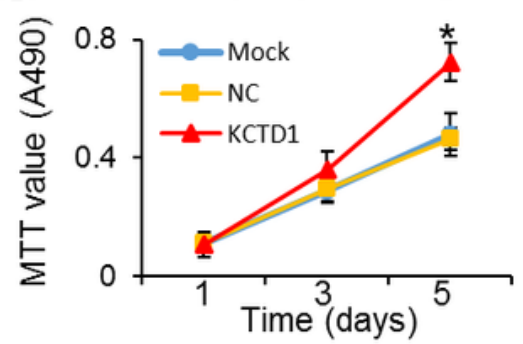

$\mathrm{F}$

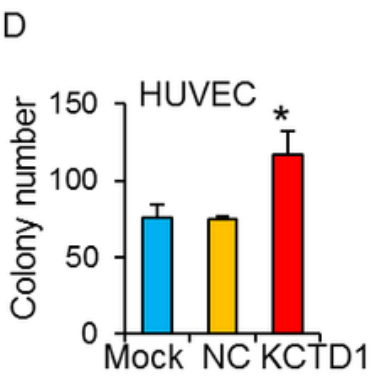

G
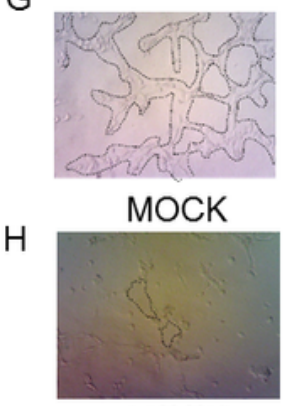

MOCK
E

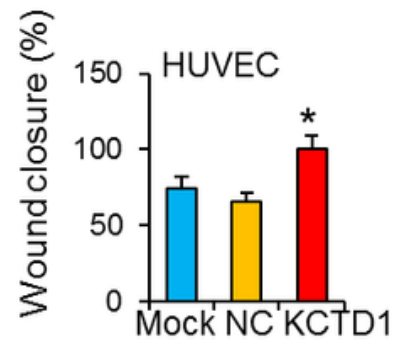

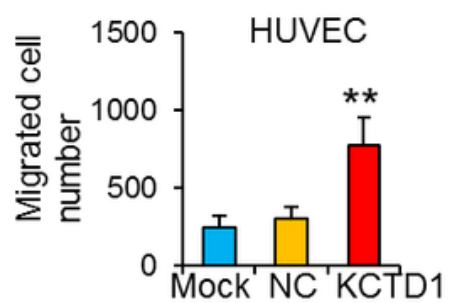

J

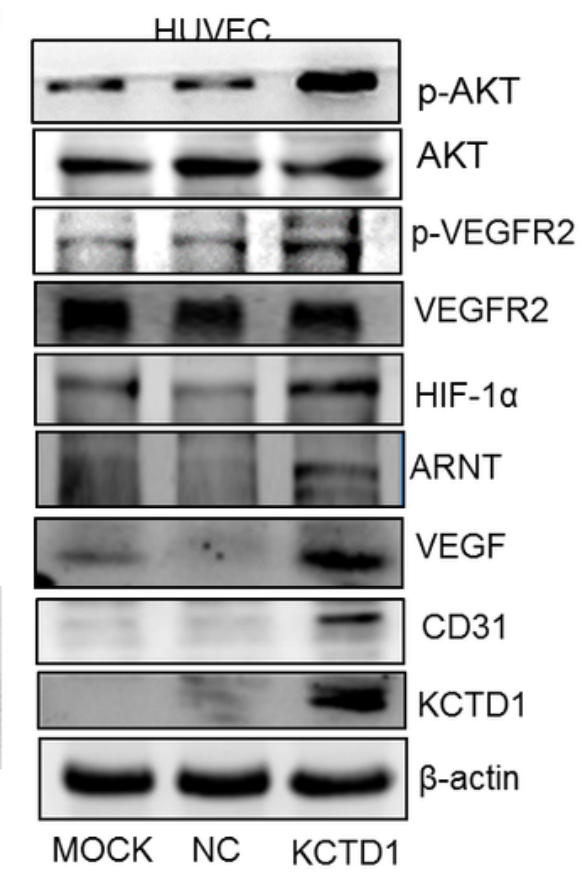

MOCK NC KCTD1
I

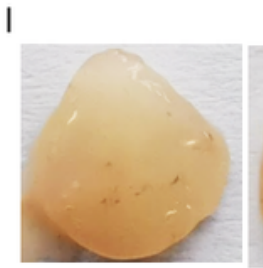

Front

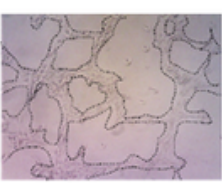

NC

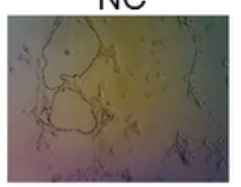

NC

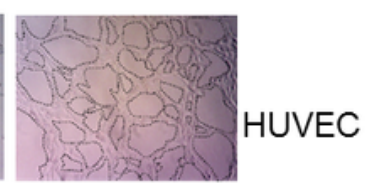

KCTD1

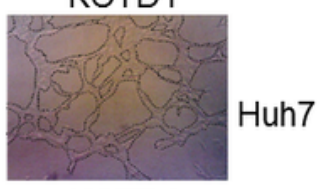

KCTD1 NC

Back

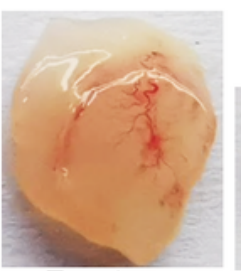

Front

KCTD1

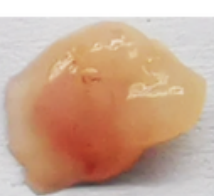

Back

\section{Figure 4}

Effects of KCTD1 overexpression on the in vitro and in vivo growth, migration and angiogenesis of HUVEC cells. a Heat map of KCTD1-overexpressing Huh7 and KCTD1-knockdown MHCC97H cells to matched control cells. The expression ratios log2(differentially expressed genes) were relative to reference genes. b Western blotting of KCTD1 expression in HUVEC cells with anti-KCTD1 antibodies. c MTT analysis of HUVEC cells infected with KCTD1-LV. 2,000 cells were seeded in a 96-well plate and 
cultured in complete DMEM medium. The absorbance at OD $490 \mathrm{~nm}$ was measured for 1-5 days. d Liquid colony formation assay of HUVEC cells infected with KCTD1-LV. 1,000 cells were plated in 6-well plates, and cultured for 12 days. The cell colonies were fixed with absolute methanol, stained with the Giemsa solution, and the number was assessed. e Scratch wound healing assays were carried out in HUVEC cells infected with KCTD1-LV. $f$ The effect of KCTD1 overexpression on HUVEC cell migration using transwell migration assays. The average number of migrated HUVEC cells across the $8-\mu \mathrm{m}$ pore-size PET membrane was shown. $g$ Tube formation capability was detected in KCTD1-overexpressing HUVECs. $h$ Tube formation analysis of HUVECs cocultured in the supernatant of KCTD1-overexpressing Huh7 cells. The tube-forming structures were circled by the black dotted line. i Growth factor reduced Matrigel including $500 \mathrm{ng} / \mathrm{ml}$ bFGF was mixed with 5×106 lentivirus-infected HUVECs and 5×106 parental Huh7 cells, which were s.c. injected into the back of immunodeficient nude mice (5-week old). 14 days later, the images of the plug were taken. $j$ Western blot results were shown to investigate the influence of KCTD1 overexpression on the VEGF signaling pathway in HUVECs. 
A

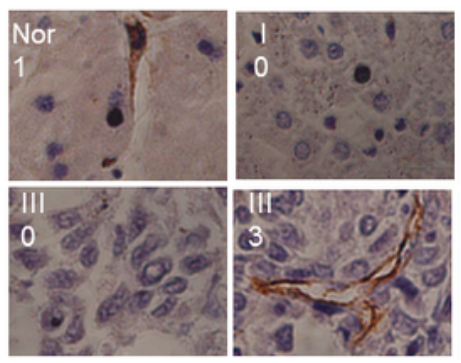

C

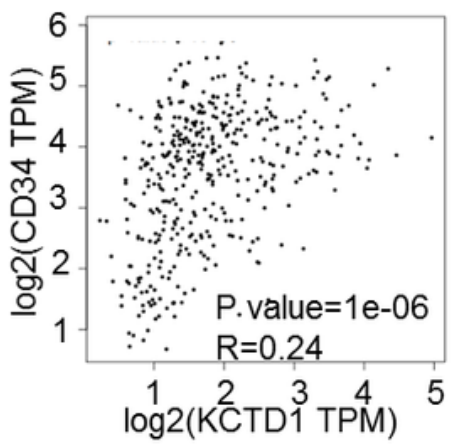

E

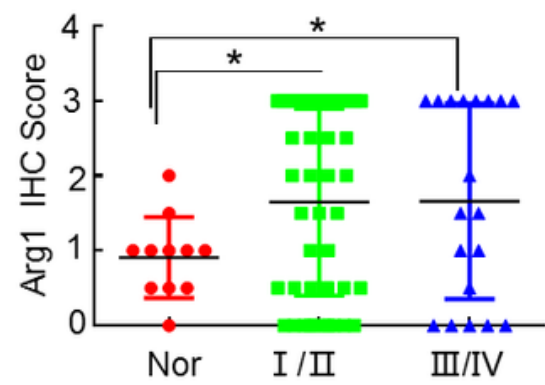

G

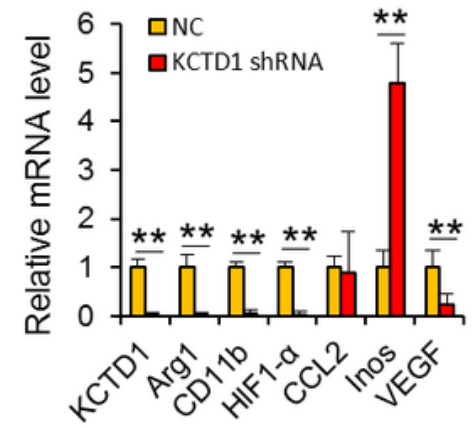

B

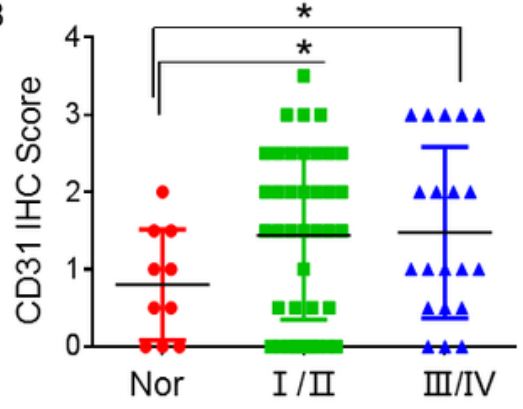

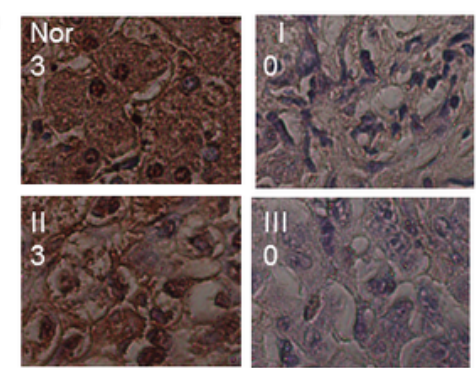

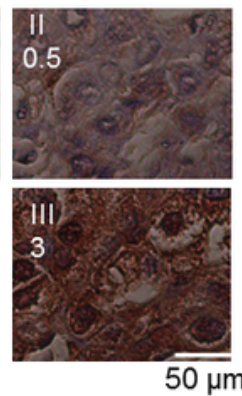

F

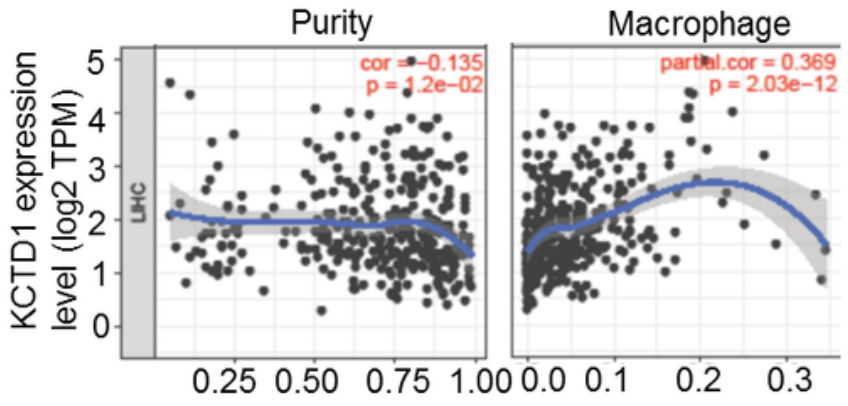

\section{Figure 5}

Expression levels of CD31 and Arg1 in hepatocellular cancer tissues. a CD31 expression detected by IHC staining in 70 HCCs and 10 non-tumor tissues. The vessel structure is stained by strong expression of CD31 (brown staining) in hepatocellular carcinoma cells. b IHC score of hepatocellular tumors and adjacent normal liver tissues detected with polyclonal anti-CD31 antibodies. The staining intensities are scored as 0-3 and a symbol indicates one individual case. Statistical comparisons of HCCs and adjacent 
normal liver tissues were carried out using the SPSS software. *, $p<0.05$; and $* *, p<0.01$. c Correlation between CD34 and KCTD1 expression analyzed in HCCs by the TCGA data. $\mathrm{d}$ Arg1 expression detected by IHC staining in 70 HCCs and 10 normal liver tissues. e Immunohistochemical score of HCCs and adjacent normal tissues detected with polyclonal anti-Arg1 antibodies. $f$ Correlation of macrophage abundance and KCTD1 in HCC determined from Timer software. $g$ The mRNA expression levels of M1 and M2 macrophage markers detected by quantitative real-time PCR in KCTD1-knockdown MHCC97H cells.

A

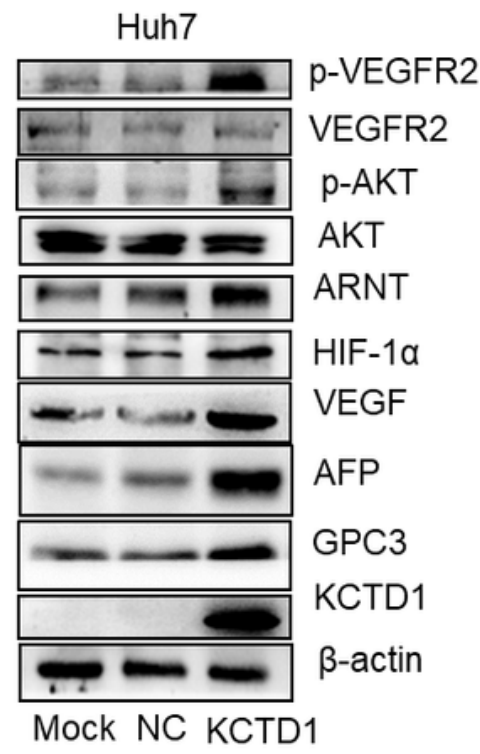

C

$$
\mathrm{MHCC} 97 \mathrm{H}
$$

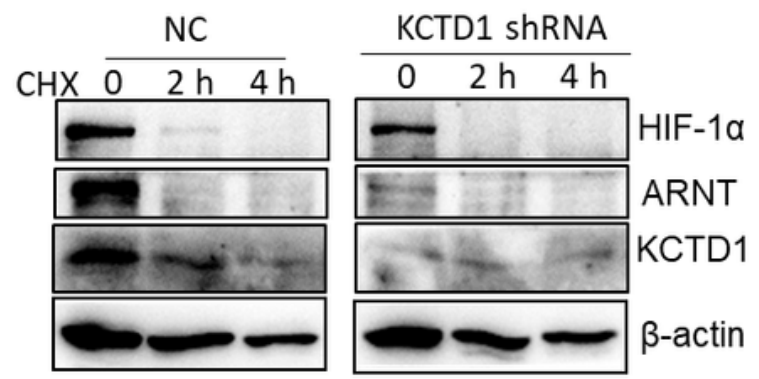

E

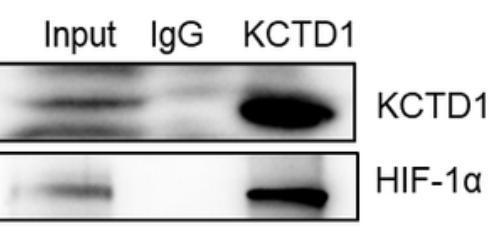

G

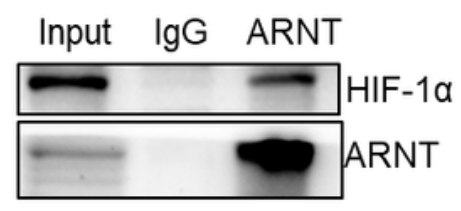

$\mathrm{H}$
B

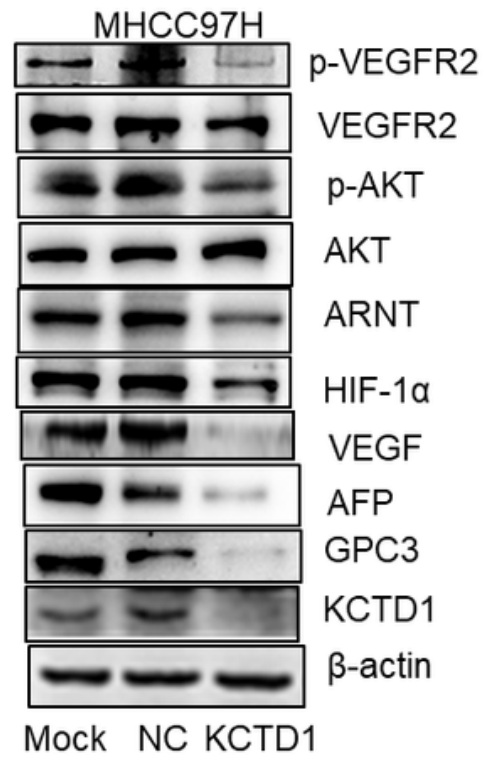

D

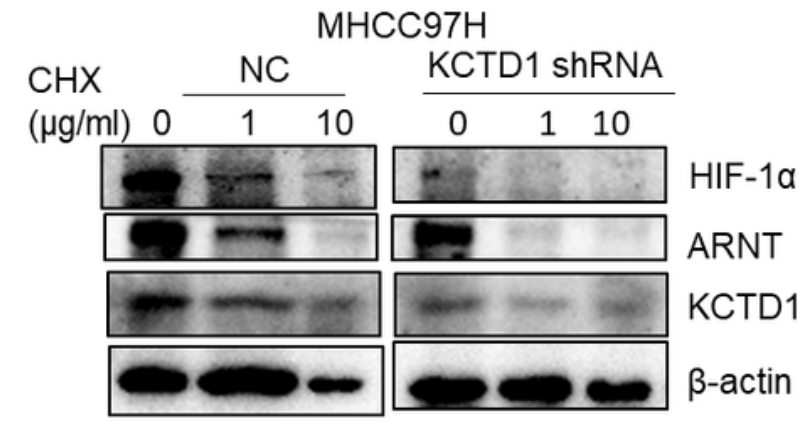

F Input IgG KCTD1
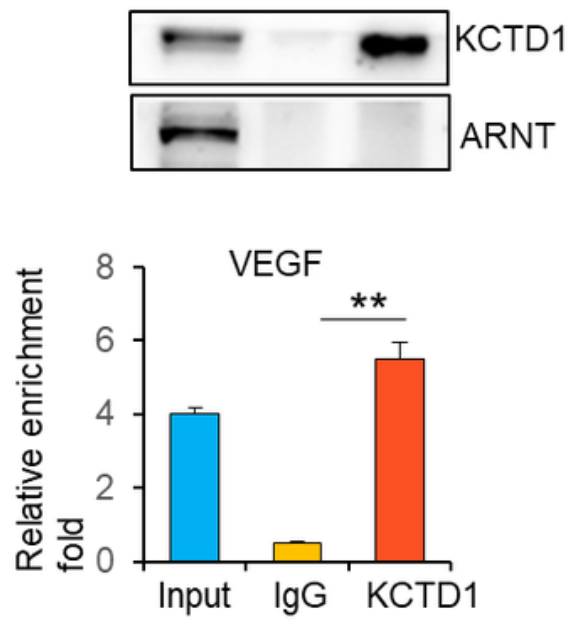

Figure 6 
Regulation mechanism of KCTD1 on the VEGF signaling pathway. a Western blots detecting the expression change of the VEGF signaling pathway in KCTD1-overexpressing Huh7 cells. b Western blot confirming the expression change of the VEGF signaling pathway in KCTD1-knockdown MHCC97H cells. c Western blots detecting the effect of KCTD1 knockdown on the degradation of HIF-1a/ARNT proteins with the treatment of $\mathrm{CHX}$ under different time points. $\mathrm{d}$ Western blots investigating the effect of KCTD1 knockdown on the degradation of HIF-1a/ARNT proteins with treatment of different concentrations of CHX. (e, f, g) Co-immunoprecipitation analysis performed to demonstrate the interaction between KCTD1 and HIF-1a, KCTD1 and ARNT or HIF-1 $a$ and ARNT. h Chromatin immunoprecipitation performed to confirm the binding of KCTD1 with the VEGF promoter. 
A

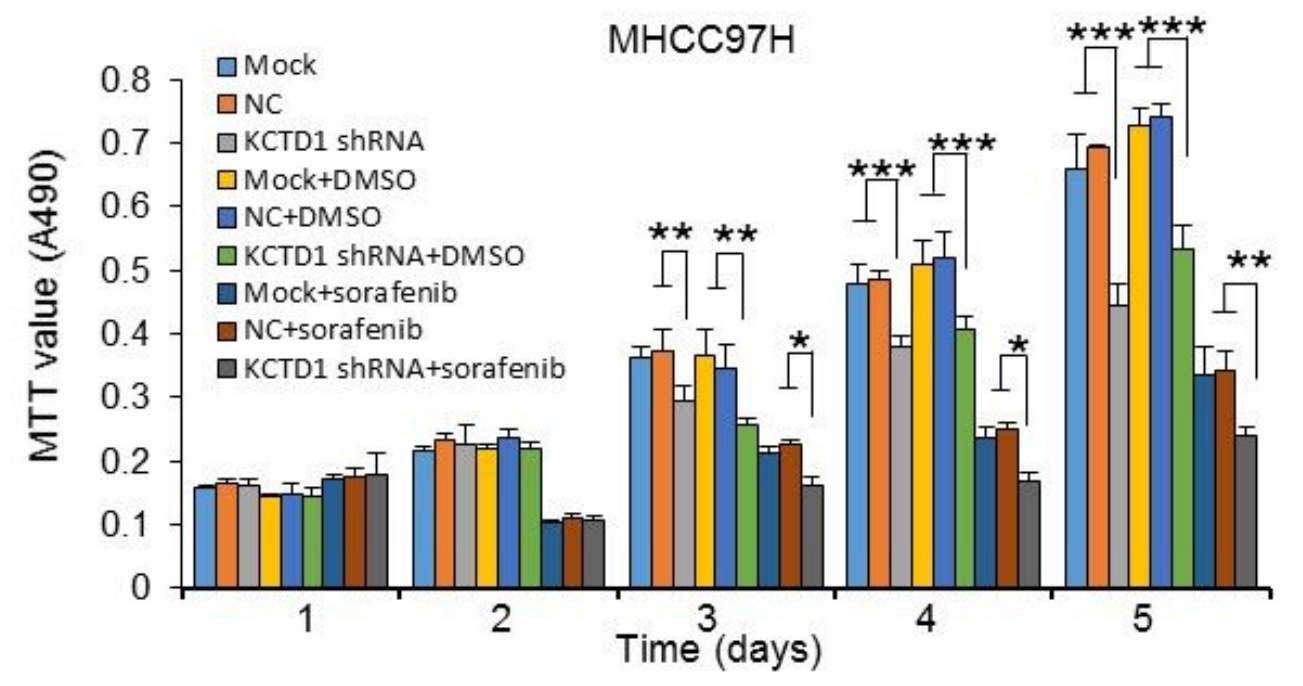

B

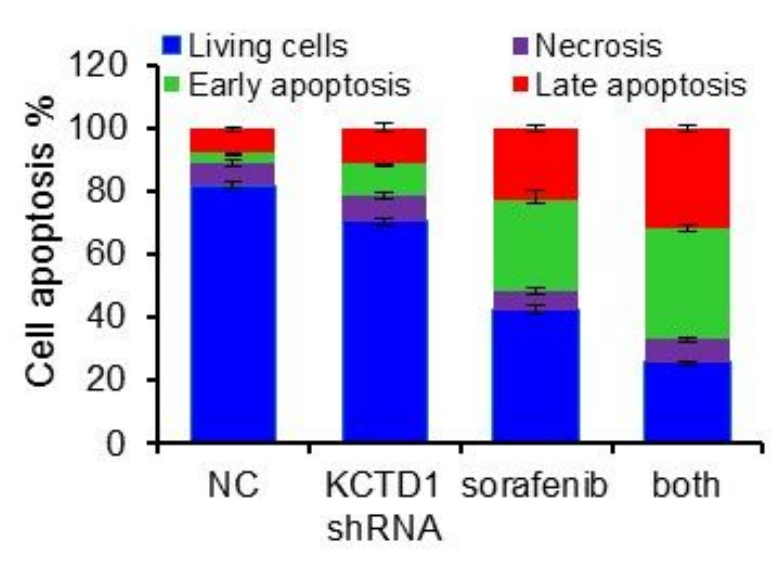

C

$\mathrm{MHCC97H}$

D

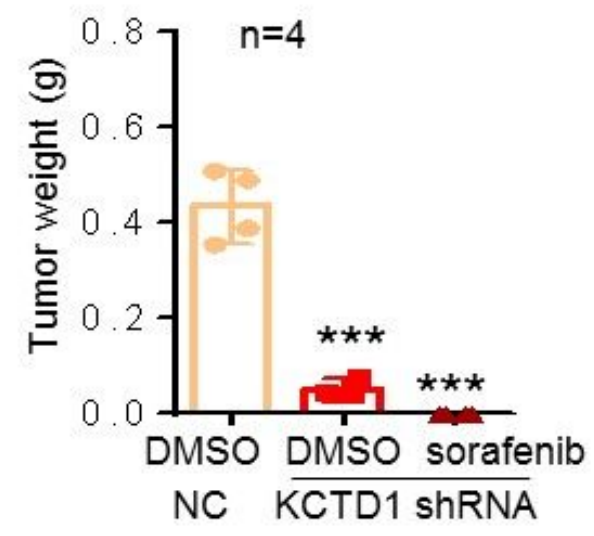

E
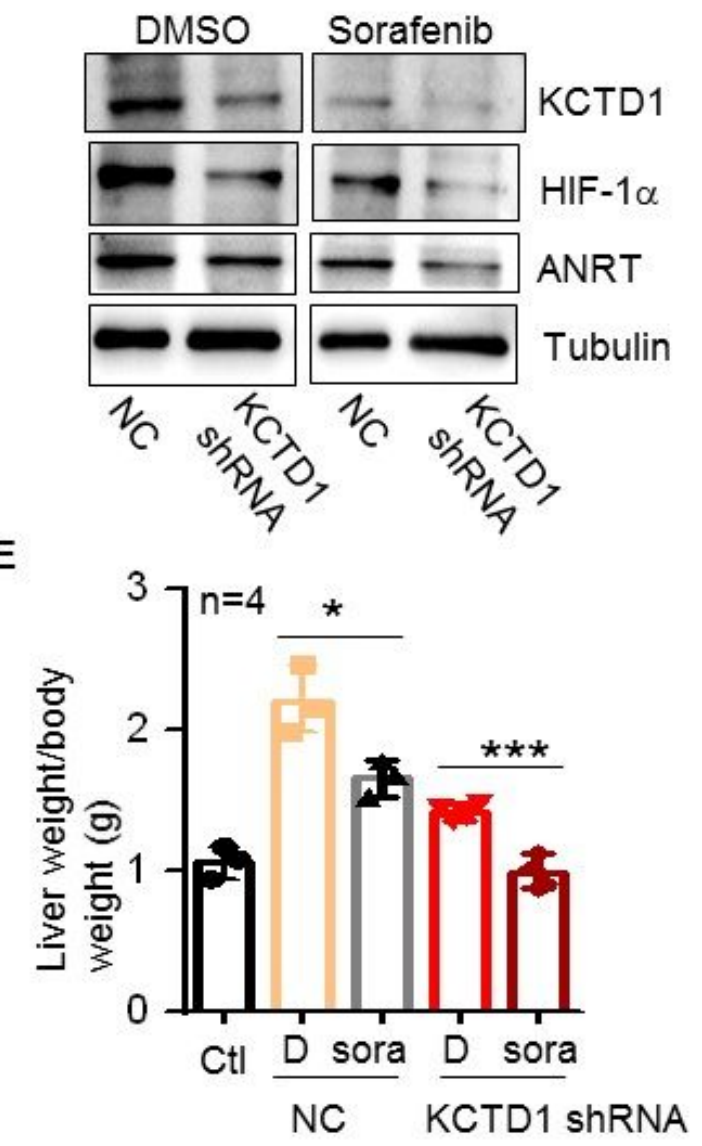

Figure 7

KCTD1 knockdown increasing the sensitivity of HCC to sorafenib. a Uninfected and infected MHCC97H cells treated with $5 \mu \mathrm{M}$ of sorafenib or DMSO for $24 \mathrm{~h}$ and cell viability assays analyzed by MTT (mean \pm SD of triplicate experiments). b Apoptosis assays of HCC cells for Flow Cytometry. MHCC97H cells infected with KCTD1 shRNA-LV are treated with $5 \mu \mathrm{M}$ of sorafenib or control agent DMSO for $24 \mathrm{~h}$ and cellular apoptosis is detected by FACS analysis. c Western blotting of the influences of sorafenib on the 
expression of KCTD1, HIF-1a/ARNT in MHCC97H cells. d About 8×106 lentivirus-infected and sorafenib treated $\mathrm{MHCC} 97 \mathrm{H}$ cells injected subcutaneously into the two points of the back of immunodeficient nude mice (female, $\mathrm{n}=4$ per group). 15 days later, the subcutaneous tumors are removed and weighed and the mean values of tumor weight are obtained in triplicate. e Intrahepatic tumor model generated by injecting $2 \times 105$ MHCC $97 \mathrm{H}$ cells. 7 days later, the mice are orally received with the control solvent or $25 \mathrm{mg} / \mathrm{kg}$ sorafenib every other day for 2 weeks and sacrificed. The tumor-loaded livers are dissected and shown. Results are presented as the mean \pm SD $(n=3)$.

A

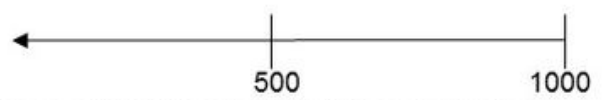

Human KCTD1 gene 3'UTR length 832 bp

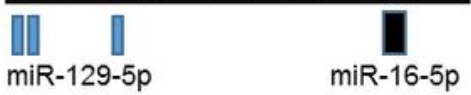

B

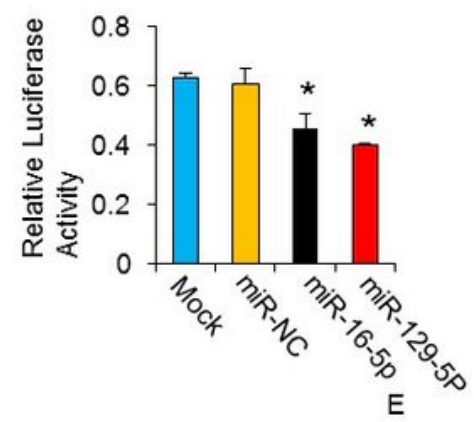

C

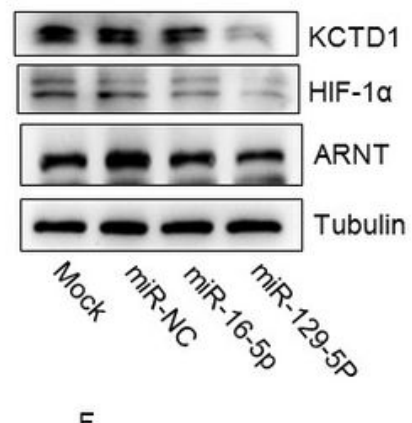

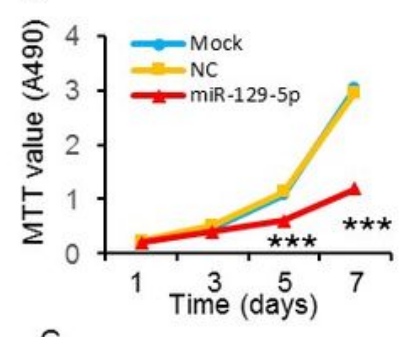

G

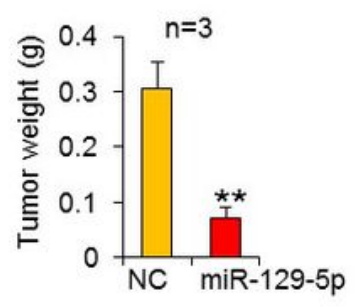

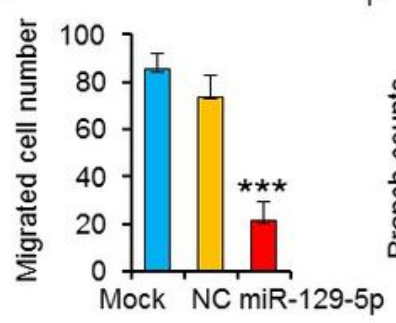

$\mathrm{H}$
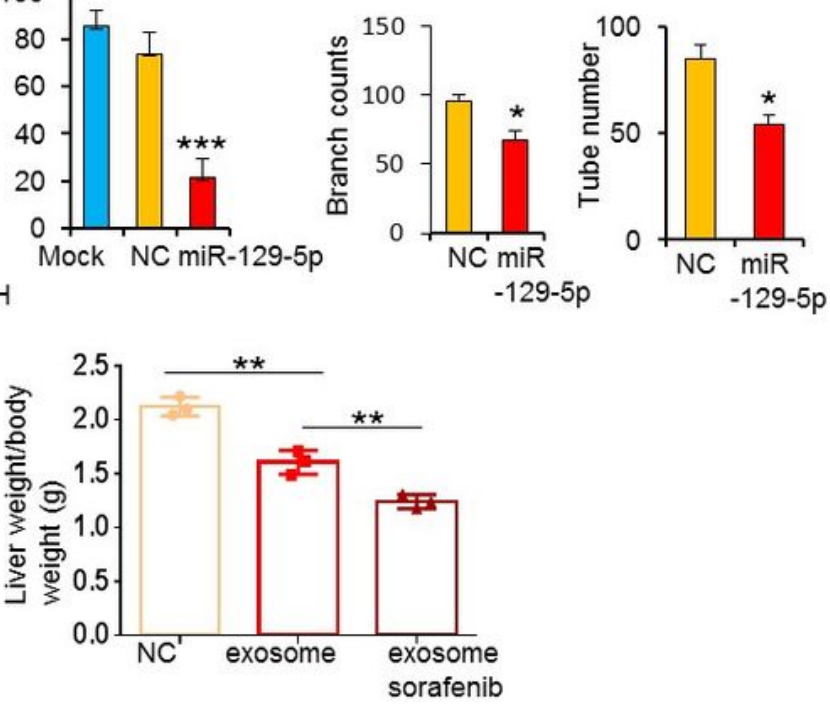

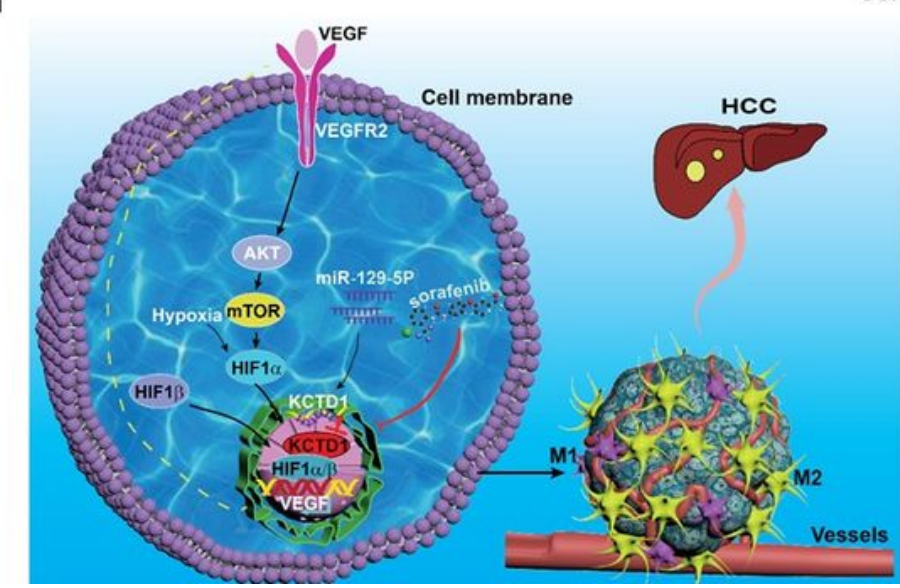




\section{Figure 8}

miR-129-5p targeting KCTD1 and reversing the effects of KCTD1 in MHCC97H cells. a Possible binding sites of miR-129-5p predicted by TargetScan in the 3'-UTR of KCTD1. b miR-129-5p regulating KCTD1 3'UTR luciferase activity. The cotransfection of miR-129-5p mimics markedly decreases the firefly luciferase reporter activity of pmirGLO-KCTD1 vector containing the KCTD1 3'-UTR. The firefly luciferase activity is normalized to the Renilla background. c Western blotting of the KCTD1 expression after miR129-5p transfection into HEK293 cells. These cells are harvested $48 \mathrm{~h}$ after miR-129-5p transfection. $\mathrm{d}$ MTT analysis of miR-129-5p infected MHCC97H cells. e Influences of miR-129-5p overexpression on MHCC97H cell migration. $f$ Tube formation analyzed by HUVEC cultured with the supernatant of miR-129$5 p$-infected MHCC97H cells. g About $8 \times 106$ infected MHCC97H cells injected subcutaneously into the two points of the back of BALb/c nude mice (4-week old, female, $n=3$ per group). 15 days later, the subcutaneous tumors are removed, weighed, and photographed. The mean weights of subcutaneous tumors are obtained in triplicate. $\mathrm{h}$ MHCC97H cells $(2 \times 106)$ injected into the liver of immunodeficient nude female mice. After one week, the mice are orally treated with the control solvent or $25 \mathrm{mg} / \mathrm{kg}$ sorafenib every other day for 3 weeks and $45 \mu \mathrm{g}$ of exosomes per mouse three times and sacrificed. The tumor-loaded livers are dissected and indicated in three groups (the control group, the exosome group, and both exosome and sorafenib group). i Schematic representation of the possible mechanism underlying KCTD1-enhanced HCC angiogenesis. KCTD1 interacts with HIF-1a/ $\beta$ in the nucleus, binds with the promoter of the VEGF gene, and activates VEGF expression, subsequently binding with VEGFR2 on the HCC cell surface and promoting the VEGF caspase signal pathway concurrently with M2 macrophage polarization. On the one hand, miR-129-5p binds with 3'UTR of the KCTD1 gene, suppresses its protein level, and reverses its oncogenic role of KCTD1. On the other hand, sorafenib targets KCTD1, abrogates its expression, and blocks its tumor-promoting function, thus enhancing HCC cells to sorafenib sensitivity. Moreover, KCTD1 knockdown and sorafenib synergistically inhibit HCC progression.

\section{Supplementary Files}

This is a list of supplementary files associated with this preprint. Click to download.

- SupportingInformation.docx 